\title{
Robust utility maximization for a diffusion market model with misspecified coefficients
}

\author{
Revaz Tevzadze • Teimuraz Toronjadze • \\ Tamaz Uzunashvili
}

Received: 1 June 2011 / Accepted: 7 May 2012 / Published online: 13 November 2012

(C) The Author(s) 2012. This article is published with open access at Springerlink.com

\begin{abstract}
The paper studies the robust maximization of utility from terminal wealth in a diffusion financial market model. The underlying model consists of a tradable risky asset whose price is described by a diffusion process with misspecified trend and volatility coefficients, and a non-tradable asset with a known parameter. The robust functional is defined in terms of a utility function. An explicit characterization of the solution is given via the solution of the Hamilton-Jacobi-Bellman-Isaacs (HJBI) equation.
\end{abstract}

Keywords Maximin problem - Saddle point · Hamilton-Jacobi-Bellman-Isaacs equation $\cdot$ Robust utility maximization $\cdot$ Generalized control

Mathematics Subject Classification (2010) $60 \mathrm{H} 10 \cdot 60 \mathrm{H} 30 \cdot 90 \mathrm{C} 47$

JEL Classification G3 - D5

R. Tevzadze $(\bowtie) \cdot$ T. Toronjadze $\cdot$ T. Uzunashvili

Business School, Georgian American University, 8 Merab Aleksidze Street, Tbilisi 0160, Georgia e-mail: rtevzadze@gmail.com

T. Uzunashvili

e-mail: tamaz.uzunashvili@mail.com

R. Tevzadze

Georgian Technical University, 77 Kostava St., Tbilisi, Georgia

T. Toronjadze

Tbilisi State University, 2 University St., Tbilisi, Georgia

e-mail: toronj333@yahoo.com 


\section{Introduction}

The purpose of the present paper is to study the robust maximization of utility from terminal wealth in a diffusion financial market model where the trend and volatility of the asset price are uncertain.

The concept of robustness was introduced by P. Huber (see [19]) in the context of statistical estimation of an unknown distribution parameter. The essence of our approach is as follows. Suppose we need to estimate the mean of some symmetric distribution. If the estimation is based on "pure" observations, then the effective estimate is the sample mean. But if observations are contaminated by outliers, then the situation changes completely. Huber introduced the so-called gross error model (the contaminated neighborhood of a true distribution) and showed that an optimal estimate is a maximum likelihood estimate constructed for the so-called least favorable distribution. Analytically, this means that we need to solve a minimax problem analogous to the problem given by (2.1) below with the asymptotic mean square error as a risk function. In some limiting cases, an optimal estimate is a median, but not a sample mean. In mathematical finance, most approaches and settings implicitly suppose that the underlying asset model is fully specified: the parameters (trend and volatility) of the model are known. Actually, we have all the same to estimate these parameters and construct, say, confidence intervals for them. Hence we only know that a pair $(\mu, \sigma)$ belongs with high probability to a rectangle $\left[\mu_{-}, \mu_{+}\right] \times\left[\sigma_{-}, \sigma_{+}\right]$. In that case there arises a problem of construction of robust trading strategies, where an optimal strategy is the best strategy against the worst state of nature. If the risk function of the problem is the expected utility from terminal wealth, then our definition of the optimization problem (2.1) is an exact one.

In 2002, Chen and Epstein [7] introduced a continuous-time intertemporal version of a multiple-priors utility function for a Brownian filtration. In that case, beliefs are represented by a set $\mathcal{P}$ of probability measures and the utility is defined as the minimum, over the set $\mathcal{P}$, of the expected utilities. Independently, Cvitanić and Karatzas [9] studied, for a given option, the hedging strategies which minimize the expected "shortfall", i.e., the difference between the payoff and the terminal wealth. They considered the problem of determining the "worst-case" model $\tilde{Q}$, i.e., the model which maximizes a minimal shortfall risk over all possible priors $Q \in \mathcal{P}$. It was shown that under certain assumptions their maximin problem could be written as a minimax problem. In 2004, Quenez [29] studied the problem of utility maximization in an incomplete multiple-priors model where asset prices are semimartingales. This problem corresponds to a maximin problem where the maximum is taken over the set of feasible wealths $X$ (or portfolios) and the minimum over the set of priors $\mathcal{P}$. The author showed that under suitable conditions, there exists a saddle point for this problem. Moreover, Quenez developed a dual approach which consists of solving a dual minimization problem over the set of priors and supermartingale measures, and showed how the solution of the dual problem leads to a solution of the primal problem.

The above maximin problems can also be called robust optimization problems since the optimization involves an entire class $\mathcal{P}$ of possible probabilistic models and thus takes into account the model risk. Optimal investment problems for such robust 
utility functionals were considered in particular by Talay and Zheng [32], Quenez [29], Schied [30], Korn and Menkens [22], Gundel [15], Bordigoni et al. [5], Föllmer and Gundel [13], Dokuchaev [12], Hernández-Hernández and Schied [16, 17].

The majority of the relevant published work is concerned with the case where one of the parameters is known exactly. For the case of an unknown drift coefficient, the existence of a saddle point of the corresponding minimax problem was established and the characterization of an optimal strategy obtained in $[9,15,16]$. For unknown volatility coefficients, the hedging strategy was constructed in $[2-4,6,11,24,34]$.

The most difficult case is to characterize the optimal strategy of the maximin problem under uncertainty about both drift and volatility terms.

Talay and Zheng [32] applied a PDE-based approach to the minimax problem and characterized the value as a viscosity solution of the corresponding HamiltonJacobi-Bellman-Isaacs (HJBI) equation. In general, such a problem does not contain a saddle point. Moreover, in robust maximization problems, the maximin should be taken instead of the minimax used by Talay and Zheng. Recently in the work of Denis and Kervarec [10], a general problem of utility maximization encompassing the case of uncertain volatility was studied, and a duality theory for robust utility maximization in this framework was established.

During the refereeing process, we have found the preprint of Matoussi et al. [27] which is also devoted to a robust utility maximization problem. To study the exponential, power and logarithmic utility maximization, the authors use 2BSDE theory (this theory was thoroughly developed by Cheridito, Soner, Touzi, Victoir and Zhang in $[8,31])$. They obtained explicit solutions in some particular cases, which is one of the tasks of our paper, too. Despite some advantages of their approach (non-Markovian models, the existence of a saddle point, a general contingent claim), we should say that that approach is not sufficiently general for our model. Namely,

(a) only the volatility matrix is misspecified in their model. In our case both coefficients (drift and volatility) are misspecified,

(b) the volatility matrix $\sqrt{a_{t}}$ satisfies the condition $\underline{a} \leq a_{t} \leq \bar{a}$, where $\underline{a}$ and $\bar{a}$ are given matrices, which does not cover our "partially misspecified volatility" case since in our paper the matrices $a_{t}=\left(\begin{array}{cc}\sigma_{t}^{2} & \rho \sigma_{t} \\ \rho \sigma_{t} & 1\end{array}\right), \underline{a}=\left(\begin{array}{cc}\sigma_{-}^{2} & \rho \sigma_{-} \\ \rho \sigma_{-} & 1\end{array}\right)$ and $\bar{a}=\left(\begin{array}{cc}\sigma_{+}^{2} & \rho \sigma_{+} \\ \rho \sigma_{+} & 1\end{array}\right)$ are non-comparable to each other.

Moreover, in the non-Markovian case the BSDE corresponding to our problem will not be a 2BSDE (see Remark 3.5). And, besides, we cannot even get our BSDE as a particular case of the 2BSDE given in [27]. So we can conclude that [27] has little in common with our paper.

In this paper, we consider an incomplete diffusion financial market model which resembles the model considered by Schied [30], Hernández-Hernández and Schied $[16,17]$. We suppose that the market consists of a risk-free asset, a tradable risky asset with misspecified trend and volatility, and a non-tradable asset with known parameters. Differently from the approach of Quenez [29] and Schied [30], we solve the maximin problem using the HJBI equation which corresponds to the primal problem. When the trend and volatility coefficients are uncertain, such a maximin problem has no saddle point in general. We extend the set of model coefficients, i.e., carry out some "randomization", and obtain as a result a minimax problem with a saddle 
point. This makes it possible to replace the maximin problem by a minimax problem, which is easier to study using the HJBI equation properties. In particular, we have found a form of this equation that coincides with the equation derived by HernándezHernández and Schied [16] when the volatility is assumed to be known. We establish the solvability of the obtained equation in the classical sense and solve the HJBI equation explicitly for a specific drift coefficient. The saddle point (an optimal portfolio and optimal coefficients) of the considered maximin problem has been found as well. An explicit characterization of the optimal strategies of the maximin problem for the case of power and exponential utilities in terms of the solution of the HJBI equation is the main result of the paper.

To illustrate our approach, we present a simple quadratic hedging problem. Let $\left(B, B^{\perp}\right)$ be a 2-dimensional Brownian motion and denote by $F^{B}=\left(\mathcal{F}_{t}^{B}\right)_{t \in[0, T]}$, $F^{B, B^{\perp}}=\left(\mathcal{F}_{t}^{B, B^{\perp}}\right)_{t \in[0, T]}$ the augmented filtrations generated by $B$ and $\left(B, B^{\perp}\right)$, respectively. We consider a filtration $F=\left(\mathcal{F}_{t}\right)_{t \in[0, T]}$ satisfying the usual conditions and $F^{B} \subseteq F \subseteq F^{B, B^{\perp}}$. Let $H$ be a square-integrable $\mathcal{F}_{T}^{B}$-measurable random variable. Denote by $\Pi^{2}$ the set of square-integrable predictable processes with respect to the filtration $F$. Let $\mathcal{P}\left(\left[\sigma_{-}, \sigma_{+}\right]\right)$be the set of probability measures on $\left[\sigma_{-}, \sigma_{+}\right]$and $\mathcal{U}, \tilde{\mathcal{U}}$ denote the set of predictable processes with respect to the filtration $F$ with values in $\left[\sigma_{-}, \sigma_{+}\right]$and $\mathcal{P}$, respectively. We use the notation $f \cdot v$ for $\int_{\sigma_{-}}^{\sigma_{+}} f(\sigma) d \nu(\sigma), f \in C\left[\sigma_{-}, \sigma_{+}\right], v \in \mathcal{P}\left(\left[\sigma_{-}, \sigma_{+}\right]\right)$. The wealth process corresponding to a portfolio process $\pi \in \Pi^{2}$ and volatility $\sigma \in \mathcal{U}$ is defined as

$$
X_{t}(\pi, \sigma)=c+\int_{0}^{t} \pi_{s} \sigma_{s} d B_{s}
$$

The problem is to find $\pi^{*} \in \Pi^{2}$ minimizing the worst case mean-variance hedging error

$$
\max _{\sigma \in \mathcal{U}} E\left|H-X_{T}\left(\pi^{*}, \sigma\right)\right|^{2}=\min _{\pi \in \Pi^{2}} \max _{\sigma \in \mathcal{U}} E\left|H-X_{T}(\pi, \sigma)\right|^{2}
$$

Such a $\pi^{*}$ is called a robust hedging strategy.

Let us extend problem (1.2) as follows. For each $v \in \tilde{\mathcal{U}}$ we define the processes

$$
\begin{aligned}
W_{t}^{v} & =\int_{0}^{t} \frac{p \cdot v_{s}}{\sqrt{p^{2} \cdot v_{s}}} d B_{s}+\int_{0}^{t} \sqrt{1-\frac{\left(p \cdot v_{s}\right)^{2}}{p^{2} \cdot v_{s}}} d B_{s}^{\perp}, \\
W_{t}^{v, \perp} & =\int_{0}^{t} \sqrt{1-\frac{\left(p \cdot v_{s}\right)^{2}}{p^{2} \cdot v_{s}}} d B_{s}-\int_{0}^{t} \frac{p \cdot v_{s}}{\sqrt{p^{2} \cdot v_{s}}} d B_{s}^{\perp},
\end{aligned}
$$

where $p, p^{2}$ are the functions $p(\sigma)=\sigma, p^{2}(\sigma)=\sigma^{2}$, respectively. One can easily check that $\left(W^{v}, W^{v, \perp}\right)$ is also a 2-dimensional Brownian motion and the equation

$$
B_{t}=\int_{0}^{t} \frac{p \cdot v_{s}}{\sqrt{p^{2} \cdot v_{s}}} d W_{s}^{v}+\int_{0}^{t} \sqrt{1-\frac{\left(p \cdot v_{s}\right)^{2}}{p^{2} \cdot v_{s}}} d W_{s}^{v, \perp}
$$


is satisfied.

For each $\pi \in \Pi^{2}, v \in \tilde{\mathcal{U}}$ we define

$$
X_{t}(\pi, v)=c+\int_{0}^{t} \pi_{s} \sqrt{p^{2} \cdot v_{s}} d W_{s}^{v} .
$$

It is clear that $\mathcal{U} \subset \tilde{\mathcal{U}}$ and for $v \in \mathcal{U}, W^{v}=B$ and (1.1) coincides with (1.4). Hence we can consider the minimax problem

$$
\min _{\pi \in \Pi^{2}} \max _{\nu \in \tilde{\mathcal{U}}} E\left|H-X_{T}(\pi, v)\right|^{2},
$$

which is the extension of problem (1.2).

For the sake of simplicity, it is assumed that $c=E H$ and, using the stochastic integral representation

$$
\begin{aligned}
H & =E H+\int_{0}^{T} h_{t} d B_{t} \\
& =E H+\int_{0}^{T} h_{t} \frac{p \cdot v_{t}}{\sqrt{p^{2} \cdot v_{t}}} d W_{t}^{v}+\int_{0}^{T} h_{t} \sqrt{1-\frac{\left(p \cdot v_{t}\right)^{2}}{p^{2} \cdot v_{t}}} d W_{t}^{v, \perp} .
\end{aligned}
$$

Equation (1.5) is rewritten as

$$
\begin{aligned}
& \min _{\pi \in \Pi^{2}} \max _{v \in \tilde{\mathcal{U}}}\left[E \int_{0}^{T}\left|h_{t} \frac{p \cdot v_{t}}{\sqrt{p^{2} \cdot v_{t}}}-\pi_{t} \sqrt{p^{2} \cdot v_{t}}\right|^{2} d t+E \int_{0}^{T} h_{t}^{2}\left(1-\frac{\left(p \cdot v_{t}\right)^{2}}{p^{2} \cdot v_{t}}\right) d t\right] \\
& \quad=\min _{\pi \in \Pi^{2}} \max _{\nu \in \tilde{\mathcal{U}}} E \int_{0}^{T}\left[\pi_{t}^{2}\left(p^{2} \cdot v_{t}\right)-2 h_{t} \pi_{t}\left(p \cdot v_{t}\right)+h_{t}^{2}\right] d t
\end{aligned}
$$

Since for each $\pi \in \Pi^{2}$

$$
\begin{gathered}
\max _{\nu \in \tilde{\mathcal{U}}} E \int_{0}^{T}\left[\pi_{t}^{2}\left(p^{2} \cdot v_{t}\right)-2 h_{t} \pi_{t}\left(p \cdot v_{t}\right)+h_{t}^{2}\right] d t \\
=\max _{\sigma \in \mathcal{U}} E \int_{0}^{T}\left[\pi_{t}^{2} \sigma_{t}^{2}-2 h_{t} \pi_{t} \sigma_{t}+h_{t}^{2}\right] d t,
\end{gathered}
$$

we have

$$
\min _{\pi \in \Pi^{2}} \max _{\sigma \in \mathcal{U}} E\left|H-X_{T}(\pi, \sigma)\right|^{2}=\min _{\pi \in \Pi^{2}} \max _{\nu \in \tilde{\mathcal{U}}} E\left|H-X_{T}(\pi, \nu)\right|^{2} .
$$

We shall see below that this expression is strictly positive. Moreover,

$$
\max _{\sigma \in \mathcal{U}} \min _{\pi \in \Pi^{2}} E\left|H-X_{T}(\pi, \sigma)\right|^{2}=\max _{\sigma \in \mathcal{U}} \min _{\pi \in \Pi^{2}} E \int_{0}^{T}\left|h_{t}-\pi_{t} \sigma_{t}\right|^{2} d t=0 .
$$

This means that a saddle point does not exist for the problem (1.2). 
On the other hand, the function $G$ defined on $\Pi^{2} \times \tilde{\mathcal{U}}$ by

$$
G(\pi, v)=E \int_{0}^{T}\left[\pi_{t}^{2}\left(p^{2} \cdot v_{t}\right)-2 h_{t} \pi_{t}\left(p \cdot v_{t}\right)+h_{t}^{2}\right] d t
$$

is convex in $\pi$ and linear in $v$. Then by the Neumann theorem (see Theorem 8 of [1], Chap. 6), there exists a saddle point $\left(\pi^{*}, \sigma^{*}\right) \in \Pi^{2} \times \tilde{\mathcal{U}}$. Therefore we have

$$
\begin{aligned}
0= & \max _{\sigma \in \mathcal{U}} \min _{\pi \in \Pi^{2}} E\left|H-X_{T}(\pi, \sigma)\right|^{2} \\
< & \min _{\pi \in \Pi^{2}} \max _{\sigma \in \mathcal{U}} E\left|H-X_{T}(\pi, \sigma)\right|^{2}=\min _{\pi \in \Pi^{2}} \max _{v \in \tilde{\mathcal{U}}} E\left|H-X_{T}(\pi, v)\right|^{2} \\
= & G\left(\pi^{*}, v^{*}\right)=\max _{\nu \in \tilde{\mathcal{U}}} \min _{\pi \in \Pi^{2}} E\left|H-X_{T}(\pi, v)\right|^{2} \\
= & \max _{\nu \in \tilde{\mathcal{U}}} \min _{\pi \in \Pi^{2}}\left[E \int_{0}^{T}\left|h_{t} \frac{p \cdot v_{t}}{\sqrt{p^{2} \cdot v_{t}}}-\pi_{t} \sqrt{p^{2} \cdot v_{t}}\right|^{2} d t\right. \\
& \left.+\int_{0}^{T} h_{t}^{2}\left(1-\frac{\left(p \cdot v_{t}\right)^{2}}{p^{2} \cdot v_{t}}\right) d t\right] \\
= & \max _{\nu \in \tilde{\mathcal{U}}} E \int_{0}^{T} h_{t}^{2}\left(1-\frac{\left(p \cdot v_{t}\right)^{2}}{p^{2} \cdot v_{t}}\right) d t .
\end{aligned}
$$

It is easy to see that the saddle point is ${ }^{1}$

$$
v_{t}^{*}=\frac{\sigma_{-}}{\sigma_{+}+\sigma_{-}} \delta_{\sigma_{+}}+\frac{\sigma_{+}}{\sigma_{+}+\sigma_{-}} \delta_{\sigma_{-}}, \quad \pi_{t}^{*}=h_{t} \frac{p \cdot v_{t}^{*}}{p^{2} \cdot v_{t}^{*}}=\frac{2 h_{t}}{\sigma_{-}+\sigma_{+}} .
$$

Thus

$$
\min _{\pi \in \Pi^{2}} \max _{\sigma \in \mathcal{U}} E\left|H-X_{T}(\pi, \sigma)\right|^{2}=F\left(\pi^{*}, \nu^{*}\right)=\left(\frac{\sigma_{-}-\sigma_{+}}{\sigma_{-}+\sigma_{+}}\right)^{2} E \int_{0}^{T} h_{t}^{2} d t .
$$

As we see, the extension of the problem allows us to find a robust strategy and the worst case mean-variance hedging error for the original problem (1.2). In Sect. 2, we shall obtain this result by means of the HJBI equation in the case of a terminal contingent claim $H\left(B_{T}\right)$.

Notice that the problem (1.2) can also be solved directly, but in more general cases (e.g. for models with nonzero drift), such "explicit computations" are complicated and to our knowledge do not exist in the literature. The aim of this work is to show that the existence of a saddle point in the extended problem simplifies solving the original problem and enables us to find "explicit solutions".

The paper is organized as follows. In Sect. 2, we describe the model and consider the misspecified coefficients as generalized controls. Furthermore, we show the existence of a saddle point of the generalized maximin problem and derive the HJBI

\footnotetext{
${ }^{1} \delta_{a}$ denotes the Dirac measure with support at a point $a$.
} 
equation for the value function. Some examples are also discussed. In Sect. 3, we prove the solvability in the classical sense of the obtained PDE in the case of power and exponential utility and give an explicit PDE characterization of the robust maximization problem.

\section{Generalized coefficients and the existence of a saddle point}

Suppose that the financial market consists of a risk-free asset

$$
d S_{t}^{0}=r\left(Y_{t}\right) S_{t}^{0} d t
$$

with $r(y) \geq 0$ and a risky financial asset whose price is defined through the stochastic differential equation (SDE)

$$
\frac{d S_{t}}{S_{t}}=\left(\tilde{b}\left(Y_{t}\right)+\mu_{t}\right) d t+\sigma_{t} d W_{t}
$$

Here $W$ is a standard Brownian motion and $Y$ denotes an economic factor process modeled by the SDE

$$
d Y_{t}=\beta\left(Y_{t}\right) d t+\left(\rho d W_{t}+\sqrt{1-\rho^{2}} d W_{t}^{\perp}\right),
$$

for some correlation factor $\rho \in[0,1]$ and a standard Brownian motion $W^{\perp}$ which is independent of $W$. Let $\left(\mathcal{F}_{t}\right)_{t \in[0, T]}$ denote the augmented filtration generated by $W, W^{\perp}$. Denote $b=\tilde{b}-r$ and assume that

(A1) $b(y), \beta(y), r(y)$ belong to $C_{b}^{1}(\mathbb{R})$,

(A2) $b^{\prime}(y), r^{\prime}(y)$ belong to $C_{0}(\mathbb{R})$,

where $C_{b}^{1}(\mathbb{R})$ is the class of bounded continuous functions with bounded derivatives and $C_{0}(\mathbb{R})$ the class of continuous functions with compact support.

Introduce the set $\mathcal{P}(K)$ of probability distributions with support on the compact set $K=\left[\mu_{-}, \mu_{+}\right] \times\left[\sigma_{-}, \sigma_{+}\right](\mathcal{P}(K)$ is a compact metric space in the weak topology), where $0 \leq \mu_{-} \leq \mu_{+}, 0<\sigma_{-} \leq \sigma_{+}$. Let $\tilde{\mathcal{U}}_{K}$ be the set of predictable $\mathcal{P}(K)$-valued processes with respect to the filtration $\left(\mathcal{F}_{t}\right)_{t \in[0, T]}$. Such a process is usually called a generalized control in control theory [35]. We identify the set of predictable $K$-valued processes $\mathcal{U}_{K}$ with the subset of $\tilde{\mathcal{U}}_{K}$ assigning to each $\left(\mu_{t}, \sigma_{t}\right)$ from $\mathcal{U}_{K}$ the $\mathcal{P}(K)$-valued process $\delta_{\left(\mu_{t}, \sigma_{t}\right)}$.

By $\Pi^{2}$ we denote the set of predictable processes with finite $L^{2}([0, T] \times \Omega)$ norm. The objective of our economic agent is to find an optimal robust strategy for the problem

$$
\max _{\pi \in \Pi^{2}} \min _{(\mu, \sigma) \in \mathcal{U}_{K}} E U\left(X_{T}^{\mu, \sigma}(\pi), Y_{T}\right),
$$

with

$$
\begin{aligned}
d X_{t} & =r\left(Y_{t}\right) X_{t} d t+\pi_{t}\left(b\left(Y_{t}\right)+\mu_{t}\right) d t+\pi_{t} \sigma_{t} d W_{t}, \quad X_{0}^{v}=x, \\
d Y_{t} & =\beta\left(Y_{t}\right) d t+\rho d W_{t}+\sqrt{1-\rho^{2}} d W_{t}^{\perp}, \quad Y_{0}=y,
\end{aligned}
$$


where $U(x, y)$ is a continuous function defined on $\mathbb{R}^{2}$ and satisfying a quadratic growth condition.

If we denote by $f \cdot v_{t}$ the integral $\int_{K} f(\mu, \sigma) v_{t}(d \mu d \sigma)$, where $f(\mu, \sigma)$ is an arbitrary continuous function, and by $p_{\mu}, p_{\sigma}$ the functions $p_{\mu}(\mu, \sigma)=\mu, p_{\sigma}(\mu, \sigma)=\sigma$, respectively, we can consider the extended maximin problem

$$
\begin{gathered}
\max _{\pi \in \Pi^{2}} \min _{v \in \tilde{\mathcal{U}}_{K}} E U\left(X_{T}^{v}(\pi), Y_{T}^{v}\right), \\
d X_{t}^{v}=r\left(Y_{t}^{v}\right) X_{t}^{v} d t+\pi_{t}\left(b\left(Y_{t}^{v}\right)+p_{\mu} \cdot v_{t}\right) d t+\pi_{t} \sqrt{p_{\sigma}^{2} \cdot v_{t}} d W_{t}, \quad X_{0}^{v}=x, \\
d Y_{t}^{v}=\beta\left(Y_{t}^{v}\right) d t+\rho \frac{p_{\sigma} \cdot v_{t}}{\sqrt{p_{\sigma}^{2} \cdot v_{t}}} d W_{t}+\sqrt{1-\rho^{2} \frac{\left(p_{\sigma} \cdot v_{t}\right)^{2}}{p_{\sigma}^{2} \cdot v_{t}}} d W_{t}^{\perp}, \quad Y_{0}^{v}=y .
\end{gathered}
$$

As follows from results of [14], there exists a strong solution of (2.4) satisfying $E\left(\sup _{t \leq T}\left|X_{t}^{v}\right|^{2}+\sup _{t \leq T}\left|Y_{t}^{\nu}\right|^{2}\right)<\infty$ for each $(\pi, v) \in \Pi^{2} \times \tilde{\mathcal{U}}_{K}$. Notice that for $(\mu, \sigma) \in \mathcal{U}_{K}$, equation (2.4) coincides with (2.2). Our aim is to show that

$$
\max _{\pi \in \Pi^{2}} \min _{(\mu, \sigma) \in \mathcal{U}_{K}} E U\left(X_{T}^{\mu, \sigma}(\pi), Y_{T}\right)=\max _{\pi \in \Pi^{2}} \min _{v \in \tilde{\mathcal{U}}_{K}} E U\left(X_{T}^{v}(\pi), Y_{T}^{v}\right)
$$

and that the latter problem admits a saddle point $\left(\pi^{*}, \nu^{*}\right)$. It is clear that $\pi^{*}$ will then be an optimal robust strategy of the initial problem (2.1), (2.2).

The link between problems (2.1), (2.2) and (2.3), (2.4) will be discussed in Theorem 2.6 below.

Remark 2.1 Let $\mathcal{B}[0, T]$ be the Borel $\sigma$-algebra on $[0, T]$ and $\tilde{\mathcal{F}}$ some $\sigma$-algebra with $\mathcal{F}_{T} \subset \tilde{\mathcal{F}}$. Then a $\mathcal{B}[0, T] \otimes \tilde{\mathcal{F}}$-measurable process $\left(\mu_{t}, \sigma_{t}\right)$ (not necessarily adapted to $\left.\left(\mathcal{F}_{t}\right)_{t \in[0, T]}\right)$ with values in the set $K$ defines an element $v \in \tilde{\mathcal{U}}_{K}$ by the formula $P\left(\left(\mu_{t}, \sigma_{t}\right) \in B \mid \mathcal{F}_{t}\right)=v_{t}(B)$. More precisely, denoting by ${ }^{p} Y$ the predictable projection of a process $Y$ (see [25]), we have the equalities ${ }^{p} \mu_{t}=\int_{K} \mu \nu_{t}(d \mu d \sigma)$, ${ }^{p} \sigma_{t}=\int_{K} \sigma v_{t}(d \mu d \sigma)$. Hence instead of (2.4) we can write

$$
\begin{aligned}
& d X_{t}=r\left(Y_{t}\right) X_{t} d t+\pi_{t}\left(b\left(Y_{t}\right)+{ }^{p} \mu_{t}\right) d t+\pi_{t} \sqrt{p_{\sigma_{t}^{2}}^{2}} d W_{t}, \quad X_{0}=x, \\
& d Y_{t}=\beta\left(Y_{t}\right) d t+\rho \frac{{ }^{p} \sigma_{t}}{\sqrt{p} \sigma_{t}^{2}} d W_{t}+\sqrt{1-\rho^{2} \frac{\left({ }^{p} \sigma_{t}\right)^{2}}{{ }^{p} \sigma_{t}^{2}}} d W_{t}^{\perp}, \quad Y_{0}=y,
\end{aligned}
$$

where we write ${ }^{p} \sigma_{t}^{2}$ for ${ }^{p}\left(\sigma_{t}^{2}\right)$.

Remark 2.2 The main theorems of the paper are valid if instead of $\Pi^{2} \times \tilde{\mathcal{U}}_{K}$ we consider the set of Markovian strategies and coefficients, i.e., the set of Borel-measurable $\mathbb{R} \times \mathcal{P}(K)$-valued functions $(\pi(t, x, y), v(t, x, y))$ such that there exists a weak solution $(X, Y)$ of (2.5) satisfying the condition $E \int_{0}^{T} \pi_{t}^{2}\left(X_{t}, Y_{t}\right) d t<\infty$. 
Since

$$
\begin{aligned}
& \left(\begin{array}{cc}
\pi_{t} \sqrt{p_{\sigma}^{2} \cdot v_{t}} & 0 \\
\rho \frac{p_{\sigma} \cdot v_{t}}{\sqrt{p_{\sigma}^{2} \cdot v_{t}}} & \sqrt{1-\rho^{2} \frac{\left(p_{\sigma} \cdot v_{t}\right)^{2}}{p_{\sigma}^{2} \cdot v_{t}}}
\end{array}\right)\left(\begin{array}{cc}
\pi_{t} \sqrt{p_{\sigma}^{2} \cdot v_{t}} & \rho \frac{p_{\sigma} \cdot v_{t}}{\sqrt{p_{\sigma}^{2} \cdot v_{t}}} \\
0 & \sqrt{1-\rho^{2} \frac{\left(p_{\sigma} \cdot v_{t}\right)^{2}}{p_{\sigma}^{2} \cdot v_{t}}}
\end{array}\right) \\
& =\left(\begin{array}{cc}
\left(p_{\sigma}^{2} \cdot v_{t}\right) \pi_{t}^{2} & \rho\left(p_{\sigma} \cdot v_{t}\right) \pi_{t} \\
\rho\left(p_{\sigma} \cdot v_{t}\right) \pi_{t} & 1
\end{array}\right),
\end{aligned}
$$

the generator of the process $\left(X^{v}, Y^{v}\right)$ can be given by

$$
\begin{aligned}
\mathcal{L}^{\pi, v} \varphi(x, y)= & \frac{1}{2} \pi_{t}^{2}\left(p_{\sigma}^{2} \cdot v_{t}\right) \varphi_{x x}(x, y)+\rho \pi_{t}\left(p_{\sigma} \cdot v_{t}\right) \varphi_{x y}(x, y)+\frac{1}{2} \varphi_{y y}(x, y) \\
& +x r(y) \varphi_{x}(x, y)+\pi_{t} b(y) \varphi_{x}(x, y)+\pi_{t}\left(p_{\mu} \cdot v_{t}\right) \varphi_{x}(x, y) \\
& +\beta(y) \varphi_{y}(x, y)
\end{aligned}
$$

for twice differentiable functions $\varphi(x, y)$ on $\mathbb{R}^{2}$. For all $\nu \in \mathcal{P}(K), \pi \in \mathbb{R},(\mu, \sigma) \in K$ and $(x, y, p, q) \in \mathbb{R} \times \mathbb{R} \times \mathbb{R}^{2} \times \mathbb{R}^{3}$, we set

$$
\begin{aligned}
\mathcal{H}^{\pi, \mu, \sigma}(x, y, p, q)= & \frac{1}{2} \pi^{2} \sigma^{2} q_{11}+\rho \pi \sigma q_{12}+\frac{1}{2} q_{22} \\
& +x r(y) p_{1}+\pi b(y) p_{1}+\pi \mu p_{1}+\beta(y) p_{2}, \\
\mathcal{H}^{\pi, v}(x, y, p, q)= & \mathcal{H}^{\pi, \cdot, \cdot}(x, y, p, q) \cdot v
\end{aligned}
$$

and

$$
\mathcal{H}(x, y, p, q)=\max _{\pi \in \mathbb{R}} \min _{\nu \in \mathcal{P}(K)} \mathcal{H}^{\pi, v}(x, y, p, q)
$$

Proposition 2.3 For each fixed $(x, y, p, q) \in \mathbb{R} \times \mathbb{R} \times \mathbb{R}^{2} \times \mathbb{R}^{3}$ with $q_{11}<0$, the function $(\pi, v) \mapsto \mathcal{H}^{\pi, v}(x, y, p, q)$ admits a saddle point $\left(\pi^{*}, v^{*}\right)$, i.e.,

$$
\begin{aligned}
\mathcal{H}^{\pi^{*}, \nu^{*}}(x, y, p, q) & =\max _{\pi \in \mathbb{R}} \min _{\nu \in \mathcal{P}(K)} \mathcal{H}^{\pi, v}(x, y, p, q) \\
& =\min _{\nu \in \mathcal{P}(K)} \max _{\pi \in \mathbb{R}} \mathcal{H}^{\pi, v}(x, y, p, q) .
\end{aligned}
$$

Moreover,

$$
\max _{\pi \in \mathbb{R}} \min _{\nu \in \mathcal{P}(K)} \mathcal{H}^{\pi, v}(x, y, p, q)=\max _{\pi \in \mathbb{R}} \min _{(\mu, \sigma) \in K} \mathcal{H}^{\pi, \mu, \sigma}(x, y, p, q) .
$$

Proof By the Neumann theorem (see Theorem 8 of [1], Chap. 6) for each fixed tuple $(x, y, p, q)$, the function of $\pi \in \mathbb{R}$ and $\nu \in \mathcal{P}(K)$ given by

$$
(\pi, v) \mapsto \mathcal{H}^{\pi, v}(x, y, p, q)
$$

admits a saddle point $\left(\pi^{*}, \nu^{*}\right)$, i.e.,

$$
\max _{\pi \in \mathbb{R}} \min _{\nu \in \mathcal{P}(K)} \mathcal{H}^{\pi, v}(x, y, p, q)=\min _{\nu \in \mathcal{P}(K)} \max _{\pi \in \mathbb{R}} \mathcal{H}^{\pi, v}(x, y, p, q)=\mathcal{H}^{\pi^{*}, v^{*}}(x, y, p, q) .
$$


It is obvious that

$$
\pi^{*}=-\frac{b(y) p_{1}+\left(p_{\mu} \cdot v^{*}\right) p_{1}+\left(p_{\sigma} \cdot v^{*}\right) \rho q_{12}}{\left(p_{\sigma}^{2} \cdot v\right) q_{11}} .
$$

Moreover, for each continuous function $f$ on $K$,

$$
\min _{\nu \in \mathcal{P}(K)} f \cdot v=\min _{(\mu, \sigma) \in K} f(\mu, \sigma)
$$

since for $v^{*}=\arg \min _{v} f \cdot v$, we have supp $v^{*} \subseteq\left\{\left(\mu^{*}, \sigma^{*}\right) \mid f\left(\mu^{*}, \sigma^{*}\right)=\min f(\mu, \sigma)\right\}$. Hence

$$
\min _{\nu \in \mathcal{P}(K)} \mathcal{H}^{\pi, v}(x, y, p, q)=\min _{(\mu, \sigma) \in K} \mathcal{H}^{\pi, \mu, \sigma}(x, y, p, q)
$$

and (2.6) is satisfied.

Now we define the value functions

$$
\begin{aligned}
& v^{-}(t, x, y)=\max _{\pi \in \Pi^{2}} \min _{(\mu, \sigma) \in \mathcal{U}_{K}} E U\left(X_{T}^{t, x, y}(\pi, v), Y_{T}^{t, x, y}(\pi, v)\right), \\
& v^{+}(t, x, y)=\min _{(\mu, \sigma) \in \tilde{\mathcal{U}}_{K}} \max _{\pi \in \Pi^{2}} E U\left(X_{T}^{t, x, y}(\pi, v), Y_{T}^{t, x, y}(\pi, v)\right),
\end{aligned}
$$

where $\left(X_{s}^{t, x, y}(\pi, v), Y_{s}^{t, x, y}(\pi, v)\right), s \geq t$, denote the solution of (2.2) with initial condition $\left(X_{t}^{t, x, y}, Y_{t}^{t, x, y}\right)=(x, y)$. Notice that if $v_{t}=\delta_{\left(\mu_{t}, \sigma_{t}\right)}$, then $Y_{s}^{t, x, y}(\pi, v)$ does not depend on $x, \pi, v$ and we can also use the notation $Y_{s}^{t, y}$.

Since the Isaacs condition is satisfied (by virtue of Proposition 2.3), there exists, as we shall see below, a value of the differential game $v \equiv v^{+}=v^{-}$, which will be a solution of the HJBI equation

$$
\begin{aligned}
& \frac{\partial}{\partial t} v(t, x, y) \\
& \quad+\mathcal{H}\left(x, y, v_{x}(t, x, y), v_{y}(t, x, y), v_{x x}(t, x, y), v_{x y}(t, x, y), v_{y y}(t, x, y)\right)=0 \\
& v(T, x, y)=U(x, y)
\end{aligned}
$$

The latter equation can be rewritten as

$$
\begin{aligned}
& \frac{\partial}{\partial t} v(t, x, y)+\frac{1}{2} v_{y y}(t, x, y)+\beta(y) v_{y}(t, x, y)+x r(y) v_{x}(t, x, y) \\
& +\min _{v \in \mathcal{P}(K)} \max _{\pi \in \mathbb{R}}\left[\frac{1}{2}\left(p_{\sigma}^{2} \cdot v\right) v_{x x}(t, x, y) \pi^{2}+\left(p_{\sigma} \cdot v\right) \rho v_{x y}(t, x, y) \pi\right. \\
& \left.+\left(b(y)+p_{\mu} \cdot v\right) v_{x}(t, x, y) \pi\right]=0, \\
& v(T, x, y)=U(x, y) .
\end{aligned}
$$


Simplifying (2.6), we get

$$
\begin{aligned}
& \min _{v \in \mathcal{P}(K)} \max _{\pi \in \mathbb{R}}\left[\frac{1}{2}\left(p_{\sigma}^{2} \cdot v\right) q_{11} \pi^{2}+\left(p_{\sigma} \cdot v\right) \rho q_{12} \pi+b(y) p_{1} \pi+\left(p_{\mu} \cdot v\right) p_{1} \pi\right] \\
& =\min _{\nu \in \mathcal{P}(K)} \frac{\left(\left(p_{\sigma} \cdot v\right) \rho q_{12}+\left(b(y)+p_{\mu} \cdot v\right) p_{1}\right)^{2}}{-2\left(p_{\sigma}^{2} \cdot v\right) q_{11}} \\
& = \begin{cases}-\frac{p_{1}^{2}}{2 q_{11}} \min _{\nu \in \mathcal{P}(K)} \frac{\left(\left(p_{\sigma} \cdot v\right) \kappa+b(y)+p_{\mu} \cdot v\right)^{2}}{p_{\sigma}^{2} \cdot v} & \text { if } p_{1} \neq 0, \\
-\frac{\rho^{2} q_{12}^{2}}{2 \sigma_{M} q_{11}} & \text { if } p_{1}=0,\end{cases}
\end{aligned}
$$

where we suppose that $q_{11}<0$ and use the notation $\kappa=\frac{\rho q_{12}}{p_{1}}, \sigma_{M}=\frac{\sigma_{-}+\sigma_{+}}{2}$.

For the sake of simplicity, we assume in addition that

(A3) $b(y)+\mu_{-} \geq 0$ for all $y \in \mathbb{R}$.

By $\varphi(z)$ we denote the linear function of $z \in\left[-\frac{\mu_{+}}{\sigma_{-}},-\frac{\mu_{-}}{\sigma_{+}}\right]$with $\varphi\left(-\frac{\mu_{+}}{\sigma_{-}}\right)=\sigma_{-}$, $\varphi\left(-\frac{\mu_{-}}{\sigma_{+}}\right)=\sigma_{+}$. Then the pair

$$
(\ell(z), m(z))= \begin{cases}\left(\mu_{+}, \frac{\mu_{+}}{z}+\frac{\sigma_{-} \sigma_{+}}{\sigma_{M}}\right) & \text { if } z \in\left(-\infty, \frac{\mu_{+} \sigma_{M}}{\sigma_{M} \sigma_{-}-\sigma_{+} \sigma_{-}}\right], \\ \left(\mu_{+}, \sigma_{-}\right) & \text {if } z \in\left(\frac{\mu_{+} \sigma_{M}}{\sigma_{M} \sigma_{-}-\sigma_{+} \sigma_{-}},-\frac{\mu_{+}}{\sigma_{-}}\right], \\ (-z \varphi(z), \varphi(z)) & \text { if } z \in\left(-\frac{\mu_{+}}{\sigma_{-}},-\frac{\mu_{-}}{\sigma_{+}}\right], \\ \left(\mu_{-}, \sigma_{+}\right) & \text {if } z \in\left(-\frac{\mu_{-}}{\sigma_{+}}, \frac{\mu_{-} \sigma_{M}}{\sigma_{M} \sigma_{+}-\sigma_{+} \sigma_{-}}\right], \\ \left(\mu_{-}, \frac{\mu_{-}}{z}+\frac{\sigma_{-} \sigma_{+}}{\sigma_{M}}\right) & \text { if } z \in\left(\frac{\mu_{-} \sigma_{M}}{\sigma_{M} \sigma_{+}-\sigma_{+} \sigma_{-}}, \infty\right)\end{cases}
$$

is a continuous, piecewise smooth function of $z \in(-\infty, \infty)$.

Proposition 2.4 There exists $v^{*} \in \mathcal{P}(K)$ of the form $v^{*}=\alpha \delta_{\mu_{a}, \sigma_{-}}+(1-\alpha) \delta_{\mu_{a}, \sigma_{+}}$, with some $(\alpha, a) \in[0,1] \times\{-,+\}$, such that

$$
\begin{aligned}
& \min _{v \in \mathcal{P}(K)} \frac{\left(b(y)+p_{\mu} \cdot v+\kappa p_{\sigma} \cdot v\right)^{2}}{p_{\sigma}^{2} \cdot v} \\
& =\frac{\left(b(y)+p_{\mu} \cdot v^{*}+\kappa p_{\sigma} \cdot v^{*}\right)^{2}}{p_{\sigma}^{2} \cdot v^{*}} \\
& = \begin{cases}\frac{\kappa\left(2\left(b(y)+\mu_{+}\right) \sigma_{M}+\kappa \sigma_{-} \sigma_{+}\right)}{\sigma_{M}^{2}} & \text { if } \kappa \in\left(-\infty, \frac{\mu_{+} \sigma_{M}}{\sigma_{M} \sigma_{-}-\sigma_{+} \sigma_{-}}\right], \\
\frac{\left(b(y)+\mu_{+}+\kappa \sigma_{-}\right)^{2}}{\sigma_{-}^{2}} & \text { if } \kappa \in\left(\frac{\mu_{+} \sigma_{M}}{\sigma_{M} \sigma_{-}-\sigma_{+} \sigma_{-}},-\frac{\mu_{+}}{\sigma_{-}}\right], \\
0 & \text { if } \kappa \in\left(-\frac{\mu_{+}}{\sigma_{-}},-\frac{\mu_{-}}{\sigma_{+}}\right], \\
\frac{\left(b(y)+\mu_{-}+\kappa \sigma_{+}\right)^{2}}{\sigma_{+}^{2}} & \text { if } \kappa \in\left(-\frac{\mu_{-}}{\sigma_{+}}, \frac{\mu_{-} \sigma_{M}}{\sigma_{M} \sigma_{+}-\sigma_{+} \sigma_{-}}\right], \\
\frac{\kappa\left(2\left(b(y)+\mu_{-}\right) \sigma_{M}+\kappa \sigma_{-} \sigma_{+}\right)}{\sigma_{M}^{2}} & \text { if } \kappa \in\left(\frac{\mu_{-} \sigma_{M}}{\sigma_{M} \sigma_{+}-\sigma_{+} \sigma_{-}}, \infty\right)\end{cases}
\end{aligned}
$$

and $\left(p_{\mu} \cdot v^{*}, p_{\sigma} \cdot v^{*}\right)=(\ell(\kappa), m(\kappa))$, where $(\ell, m)$ is defined by $(2.9)$.

The proof is given in the Appendix. 


\section{Corollary 2.5}

$$
\begin{aligned}
\min _{v \in \mathcal{P}(K)} & \frac{\left.\left(b(y)+p_{\mu} \cdot v\right) p_{1}+\left(p_{\sigma} \cdot v\right) \rho q_{12}\right)^{2}}{-2 p_{\sigma}^{2} \cdot v q_{11}} \\
= & \min _{(\mu, \sigma) \in K} \frac{\left(b(y) p_{1}+\mu p_{1}+\sigma \rho q_{12}\right)^{2}}{-2\left(2 \sigma_{M} \sigma-\sigma_{-} \sigma_{+}\right) q_{11}} \\
= & -\frac{\rho q_{12}\left(2 p_{1}\left(b(y)+\mu_{+}\right) \sigma_{M}+\rho q_{12} \sigma_{-} \sigma_{+}\right)}{2 q_{11} \sigma_{M}^{2}} \\
& \times \chi\left(\frac{\rho q_{12}}{p_{1}} \in\left(-\infty, \frac{\mu_{+} \sigma_{M}}{\sigma_{M} \sigma_{-}-\sigma_{+} \sigma_{-}}\right]\right) \\
& -\frac{\left(p_{1}\left(b(y)+\mu_{+}\right)+\rho q_{12} \sigma_{-}\right)^{2}}{2 q_{11} \sigma_{-}^{2}} \chi\left(\frac{\rho q_{12}}{p_{1}} \in\left(\frac{\mu_{+} \sigma_{M}}{\sigma_{M} \sigma_{-}-\sigma_{+} \sigma_{-}},-\frac{\mu_{+}}{\sigma_{-}}\right]\right) \\
& -\frac{\left(p_{1}\left(b(y)+\mu_{-}\right)+\rho q_{12} \sigma_{+}\right)^{2}}{2 q_{11} \sigma_{+}^{2}} \chi\left(\frac{\rho q_{12}}{p_{1}} \in\left(-\frac{\mu_{-}}{\sigma_{+}}, \frac{\mu_{-} \sigma_{M}}{\sigma_{M} \sigma_{+}-\sigma_{+} \sigma_{-}}\right]\right) \\
& -\frac{\rho q_{12}\left(2 p_{1}\left(b(y)+\mu_{-}\right) \sigma_{M}+\rho q_{12} \sigma_{-} \sigma_{+}\right)}{2 q_{11} \sigma_{M}^{2}} \\
& \times \chi\left(\frac{\rho q_{12}}{p_{1}} \in\left(\frac{\mu-\sigma_{M}}{\sigma_{M} \sigma_{+}-\sigma_{+} \sigma_{-}}, \infty\right)\right)-\frac{\rho^{2} q_{12}^{2}}{2 \sigma_{M}} \chi\left(p_{1}=0\right),
\end{aligned}
$$

where $\chi(A)$ denotes the indicator of a set $A$.

Proof It is sufficient to verify that for $\nu_{ \pm}^{*}=\alpha \delta_{\mu_{ \pm}, \sigma_{-}}+(1-\alpha) \delta_{\mu_{ \pm}, \sigma_{+}}, 0 \leq \alpha \leq 1$, we get $p_{\sigma}^{2} \cdot v_{ \pm}^{*}=2 \sigma_{M}\left(p_{\sigma} \cdot v_{ \pm}^{*}\right)-\sigma_{-} \sigma_{+}$.

From Corollary 2.5, we obtain the result that the HJBI equation has the form

$$
\begin{aligned}
& \frac{\partial}{\partial t} v(t, x, y)+\frac{1}{2} v_{y y}(t, x, y)+\beta(y) v_{y}(t, x, y)+x r(y) v_{x}(t, x, y) \\
& \quad+\min _{(\mu, \sigma) \in K} \frac{\left(b(y) v_{x}(t, x, y)+\mu v_{x}(t, x, y)+\rho \sigma v_{x y}(t, x, y)\right)^{2}}{-2\left(2 \sigma_{M} \sigma-\sigma_{-} \sigma_{+}\right) v_{x x}(t, x, y)}=0 \\
& v(T, x, y)=U(x, y)
\end{aligned}
$$

A classical solution $v(t, x, y)$ of this equation defines a pair of continuous, piecewise smooth functions of $(t, x, y)$,

$$
(\bar{\ell}(t, x, y), \bar{m}(t, x, y))=\left(\ell\left(\frac{\rho v_{x y}(t, x, y)}{v_{x}(t, x, y)}\right), m\left(\frac{\rho v_{x y}(t, x, y)}{v_{x}(t, x, y)}\right)\right),
$$

by the formula (2.9).

The following theorem is the verification theorem of [28] adapted to our setting. 
Theorem 2.6 (Verification theorem) Let $v(t, x, y)$ be a classical solution of (2.7), (2.8) such that $v_{x x}(t, x, y)<0$ and for some constants $L>0, p \geq 1$,

$$
\begin{aligned}
|v(t, x, y)| & \leq L(1+|x|+|y|)^{p}, \\
\left|\frac{v_{x}(t, x, y)}{v_{x x}(t, x, y)}\right| & \leq L(1+|x|+|y|), \\
\left|\frac{v_{x y}(t, x, y)}{v_{x x}(t, x, y)}\right| & \leq L(1+|x|+|y|) .
\end{aligned}
$$

Suppose also that the triplet $\left(\pi^{*}(t, x, y), p_{\mu} \cdot v^{*}(t, x, y), p_{\sigma} \cdot v^{*}(t, x, y)\right)$ satisfies a Lipschitz condition on each compact subset of $[0, T] \times \mathbb{R} \times \mathbb{R}$, where

$$
\begin{aligned}
\pi^{*}(t, x, y) & \\
=- & \frac{\left(b(y)+p_{\mu} \cdot v^{*}(t, x, y)\right) v_{x}(t, x, y)+p_{\sigma} \cdot v^{*}(t, x, y) \rho v_{x y}(t, x, y)}{\left(2 \sigma_{M} p_{\sigma} \cdot v^{*}(t, x, y)-\sigma_{-} \sigma_{+}\right) v_{x x}(t, x, y)},
\end{aligned}
$$

and where $\left(p_{\mu} \cdot v^{*}(t, x, y), p_{\sigma} \cdot v^{*}(t, x, y)\right)$ coincides with $(\bar{\ell}(t, x, y), \bar{m}(t, x, y))$ defined by (2.12). Then $\left(\pi^{*}, v^{*}\right)$ is a saddle point of the problem (2.3), (2.4) and

$$
\begin{aligned}
\max _{\pi \in \Pi^{2}} \min _{(\mu, \sigma) \in \mathcal{U}_{K}} E U\left(X_{T}^{\mu, \sigma}(\pi), Y_{T}\right) & =\max _{\pi \in \Pi^{2}} \min _{\nu \in \tilde{\mathcal{U}}_{K}} E U\left(X_{T}^{v}(\pi), Y_{T}^{v}\right) \\
& =\min _{\nu \in \tilde{\mathcal{U}}_{K}} \max _{\pi \in \Pi^{2}} E U\left(X_{T}^{v}(\pi), Y_{T}^{v}\right) .
\end{aligned}
$$

Proof By the definition of (2.14), (2.12), the pair $\left(\pi^{*}(t, x, y), v^{*}(t, x, y)\right)$ is a saddle point of the function

$$
\begin{aligned}
f(t, x, y, \pi, v)= & \frac{1}{2}\left(p_{\sigma}^{2} \cdot v\right) v_{x x}(t, x, y) \pi^{2}+\left(p_{\sigma} \cdot v\right) \rho v_{x y}(t, x, y) \pi \\
& +\left(b(y)+p_{\mu} \cdot v\right) v_{x}(t, x, y) \pi
\end{aligned}
$$

for each $(t, x, y)$. It is easy to see that this pair is a continuous, piecewise smooth function of variables $(t, x, y) \in[0, T] \times \mathbb{R} \times \mathbb{R}$. By definition, the triplet of functions $\left(\pi^{*}(t, x, y), p_{\mu} \cdot v^{*}(t, x, y), p_{\sigma} \cdot v^{*}(t, x, y)\right)$ consists of Lipschitz functions on each compact subset. Since $p_{\sigma}^{2} \cdot v^{*}(t, x, y)=2 \sigma_{M}\left(p_{\sigma} \cdot v^{*}(t, x, y)\right)-\sigma_{-} \sigma_{+} \geq \sigma_{-}^{2}$ is satisfied, $\frac{1}{p_{\sigma}^{2} \cdot v^{*}(t, x, y)}$ is also a Lipschitz function on each compact subset. The linear growth condition for the triplet is also satisfied since $\left|p_{\mu} \cdot v^{*}(t, x, y)\right| \leq \mu_{+}$, $\sigma_{-} \leq\left|p_{\sigma} \cdot v^{*}(t, x, y)\right| \leq \sigma_{+}$and the inequalities

$$
\begin{aligned}
\left|\pi^{*}(t, x, y)\right| & =\left|\frac{\left(b(y)+p_{\mu} \cdot v^{*}(t, x, y)\right) v_{x}(t, x, y)+p_{\sigma} \cdot v^{*}(t, x, y) \rho v_{x y}(t, x, y)}{\left(2 \sigma_{M} p_{\sigma} \cdot v^{*}(t, x, y)-\sigma_{-} \sigma_{+}\right) v_{x x}(t, x, y)}\right| \\
& \leq \frac{\max _{y}\left(b(y)+\mu_{+}\right)}{\sigma_{-}^{2}}\left|\frac{v_{x}(t, x, y)}{v_{x x}(t, x, y)}\right|+\frac{\rho \sigma_{+}}{\sigma_{-}^{2}}\left|\frac{v_{x y}(t, x, y)}{v_{x x}(t, x, y)}\right| \\
& \leq \bar{L}(1+|x|+|y|)
\end{aligned}
$$


hold for some constant $\bar{L}$ thanks to condition (2.13). Thus the SDE

$$
\begin{aligned}
d X_{t}^{*}= & r\left(Y_{t}^{*}\right) X_{t}^{*} d t+\pi^{*}\left(t, X_{t}^{*}, Y_{t}^{*}\right)\left(b\left(Y_{t}^{*}\right)+p_{\mu} \cdot v^{*}\left(t, X^{*}, Y^{*}\right)\right) d t \\
& +\pi^{*}\left(t, X_{t}^{*}, Y_{t}^{*}\right) \sqrt{p_{\sigma}^{2} \cdot v^{*}\left(t, X_{t}^{*}, Y_{t}^{*}\right)} d W_{t}, \\
X_{0}^{*}= & x, \\
d Y_{t}^{*}= & \beta\left(Y_{t}^{*}\right) d t+\rho \frac{p_{\sigma} \cdot v^{*}\left(t, X_{t}^{*}, Y_{t}^{*}\right)}{\sqrt{p_{\sigma}^{2} \cdot v^{*}\left(t, X_{t}^{*}, Y_{t}^{*}\right)}} d W_{t} \\
& +\sqrt{1-\rho^{2} \frac{p_{\sigma} \cdot v^{*}\left(t, X_{t}^{*}, Y_{t}^{*}\right)^{2}}{p_{\sigma}^{2} \cdot v^{*}\left(t, X_{t}^{*}, Y_{t}^{*}\right)}} d W_{t}^{\perp}, \\
Y_{0}^{*}= & y,
\end{aligned}
$$

defining an optimal wealth process has coefficients which are Lipschitz functions on each set $\{(t, x, y):|x| \leq R,|y| \leq R\}$ and satisfy a linear growth condition. Hence there exists a unique strong solution of that SDE with the properties $E \sup _{t \leq T}\left|X_{t}^{*}\right|^{k}<\infty, E \sup _{t \leq T}\left|Y_{t}^{*}\right|^{k}<\infty$, for each $k \geq 1$ (see Theorem V.2.3 of [14]), and also satisfying $E \int_{0}^{T} \pi^{* 2}\left(t, X^{*}, Y^{*}\right) d t<\infty$. We denote by $\left(X_{s}^{* t, x, y}, Y_{s}^{* t, x, y}\right), s \geq t$, the solution of the system (2.4) corresponding to $\pi^{*}, v^{*}$ with initial condition $\left(X_{t}^{* t, x, y}, Y_{t}^{* t, x, y}\right)=(x, y)$.

Let $\tau_{R}^{t, x, y}=T \wedge \inf \left\{s \geq t:\left|X_{s}^{* t, x, y}\right| \geq R,\left|Y_{s}^{* t, x, y}\right| \geq R\right\}$. Since

$$
\begin{aligned}
\frac{\partial}{\partial t} v & +\mathcal{H}^{\pi^{*}, v^{*}}\left(x, y, v_{x}, v_{y}, v_{x x}, v_{x y}, v_{y y}\right) \\
& =\frac{\partial}{\partial t} v+\frac{1}{2} v_{y y}+\beta(y) v_{y}+x r(y) v_{x}+f\left(t, x, y, \pi^{*}, \cdot\right) \cdot v^{*}=0
\end{aligned}
$$

and $v_{x} \pi^{*}, v_{y}$ are continuous bounded functions on each ball, we can apply Itô's formula to $v\left(s, X_{s}^{*}, Y_{s}^{*}\right)$ and get $v(t, x, y)=E v\left(X_{\tau_{R}^{t, x, y}}^{* t, x, y}, Y_{\tau_{R}^{t, x, y}}^{* t, x, y}\right)$. Passing to the limit as $R \rightarrow \infty$, we obtain

$$
v(t, x, y)=E U\left(X_{T}^{* t, x, y}, Y_{T}^{* t, x, y}\right),
$$

since by the integrability of $\sup _{t \leq T}\left|X_{t}^{*}\right|^{p}+\sup _{t \leq T}\left|Y_{t}^{*}\right|^{p}$, we have

$$
P\left(\tau_{R}^{t, x, y}<T\right) \leq P\left(\sup _{t \leq T}\left|X_{t}^{*}\right| \geq R, \sup _{t \leq T}\left|Y_{t}^{*}\right| \geq R\right) \longrightarrow 0 \quad \text { as } R \rightarrow \infty
$$

and

$$
\begin{aligned}
& \left|E v\left(X_{T}^{* t, x, y}, Y_{T}^{* t, x, y}\right)-E v\left(\tau_{R}^{t, x, y}, X_{\tau_{R}^{t, x, y}}^{* t, x, y}, Y_{\tau_{R}^{t, x, y}}^{* t, x, y}\right)\right| \\
& \quad \leq\left|E v\left(X_{T}^{* t, x, y}, Y_{T}^{* t, x, y}\right) \chi\left(\tau_{R}^{t, x, y}<T\right)\right|+\left|E v\left(X_{\tau_{R}^{t, x, y}}^{* t, x, y}, Y_{\tau_{R}^{t, x, y}}^{* t, x, y}\right) \chi\left(\tau_{R}^{t, x, y}<T\right)\right| \\
& \quad \leq 2 L E\left(1+\sup _{t \leq T}\left|X_{t}^{*}\right|^{p}+\sup _{t \leq T}\left|Y_{t}^{*}\right|^{p}\right) \chi\left(\tau_{R}^{t, x, y}<T\right) \longrightarrow 0 \quad \text { as } R \rightarrow \infty .
\end{aligned}
$$


Similarly, using Itô's formula for the processes $v\left(s, X_{s}^{* t, x, y}\left(\pi^{*}, v\right), Y_{s}^{* t, x, y}\left(\pi^{*}, v\right)\right)$ and $v\left(s, X_{s}^{* t, x, y}\left(\pi, v^{*}\right), Y_{s}^{* t, x, y}\left(\pi, v^{*}\right)\right)$ and taking into account the inequalities

$$
\begin{aligned}
f(t, x, y, \pi, \cdot) \cdot v^{*}(t, x, y) & \leq f\left(t, x, y, \pi^{*}(t, x, y), \cdot\right) \cdot v^{*}(t, x, y) \\
& \leq f\left(t, x, y, \pi^{*}(t, x, y), \cdot\right) \cdot v,
\end{aligned}
$$

we get

$$
E U\left(X_{T}^{t, x, y}\left(\pi, v^{*}\right), Y_{T}^{t, x, y}\left(\pi, v^{*}\right)\right) \leq v(t, x, y) \leq E U\left(X_{T}^{t, x, y}\left(\pi^{*}, \nu\right), Y_{T}^{t, x, y}\left(\pi^{*}, v\right)\right) .
$$

Finally, we obtain

$$
\begin{aligned}
E U\left(X_{T}^{t, x, y}\left(\pi, v^{*}\right), Y_{T}^{t, x, y}\left(\pi, v^{*}\right)\right) & \leq E U\left(X_{T}^{* t, x, y}, Y_{T}^{* t, x, y}\right) \\
& \leq E U\left(X_{T}^{t, x, y}\left(\pi^{*}, v\right), Y_{T}^{t, x, y}\left(\pi^{*}, v\right)\right) .
\end{aligned}
$$

This means that the pair $\left(\pi^{*}, v^{*}\right)$ is a saddle point of problem (2.3).

Since $v(t, x, y)=\inf _{v \in \tilde{\mathcal{U}}_{K}} E U\left(X_{T}^{t, x, y}\left(\pi^{*}, v\right), Y_{T}^{t, x, y}\left(\pi^{*}, v\right)\right)$ satisfies the HJB equation of the stochastic control problem $\min _{v \in \tilde{\mathcal{U}}_{K}} E U\left(X_{T}^{v}\left(\pi^{*}\right), Y_{T}^{v}\right)$ for

$$
\begin{aligned}
d X_{t}^{v}= & r\left(Y_{t}^{v}\right) X_{t}^{v} d t+\pi_{t}^{*}\left(X_{t}^{v}, Y_{t}^{v}\right)\left(b\left(Y_{t}^{v}\right)+p_{\mu} \cdot v_{t}\right) d t \\
& +\pi_{t}^{*}\left(X_{t}^{v}, Y_{t}^{v}\right) \sqrt{p_{\sigma}^{2} \cdot v_{t}} d W_{t}, \quad X_{0}^{v}=x \\
d Y_{t}^{v}= & \beta\left(Y_{t}^{v}\right) d t+\rho \frac{p_{\sigma} \cdot v_{t}}{\sqrt{p_{\sigma}^{2} \cdot v_{t}}} d W_{t}+\sqrt{1-\rho^{2} \frac{\left(p_{\sigma} \cdot v_{t}\right)^{2}}{p_{\sigma}^{2} \cdot v_{t}}} d W_{t}^{\perp}, \quad Y_{0}^{v}=y
\end{aligned}
$$

and

$$
\min _{\nu \in \mathcal{P}(K)} f\left(t, x, y, \pi^{*}(t, x, y), \cdot\right) \cdot v=\min _{(\mu, \sigma) \in K} f\left(t, x, y, \pi^{*}(t, x, y), \mu, \sigma\right),
$$

we conclude that

$$
\begin{aligned}
v(t, x, y) & =\min _{\nu \in \tilde{\mathcal{U}}_{K}} \operatorname{EU}\left(X_{T}^{t, x, y}\left(\pi^{*}, v\right), Y_{T}^{t, x, y}\left(\pi^{*}, v\right)\right) \\
& =\min _{(\mu, \sigma) \in \mathcal{U}_{K}} \operatorname{EU}\left(X_{T}^{t, x, y}\left(\pi^{*}, \mu, \sigma\right), Y_{T}^{t, x, y}(\pi, \mu, \sigma)\right) .
\end{aligned}
$$

Thus

$$
\begin{aligned}
& \min _{\nu \in \tilde{\mathcal{U}}_{K}} \max _{\pi \in \Pi^{2}} E U\left(X_{T}^{t, x, y}(\pi, \nu), Y_{T}^{t, x, y}(\pi, \nu)\right) \\
& \quad \leq \max _{\pi \in \Pi^{2}} E U\left(X_{T}^{t, x, y}\left(\pi, \nu^{*}\right), Y_{T}^{t, x, y}\left(\pi, v^{*}\right)\right) \\
& \quad \leq v(t, x, y) \\
& \quad=\min _{(\mu, \sigma) \in \mathcal{U}_{K}} E U\left(X_{T}^{t, x, y}\left(\pi^{*}, \mu, \sigma\right), Y_{T}^{t, x, y}(\pi, \mu, \sigma)\right) \\
& \quad \leq \max _{\pi \in \Pi^{2}} \min _{(\mu, \sigma) \in \mathcal{U}_{K}} E U\left(X_{T}^{t, x, y}(\pi, \mu, \sigma), Y_{T}^{t, x, y}(\pi, \mu, \sigma)\right) .
\end{aligned}
$$


On the other hand,

$$
\begin{gathered}
\max _{\pi \in \Pi^{2}} \min _{(\mu, \sigma) \in \mathcal{U}_{K}} E U\left(X_{T}^{t, x, y}(\pi, \mu, \sigma), Y_{T}^{t, x, y}(\pi, \mu, \sigma)\right) \\
\quad \leq \min _{\nu \in \tilde{\mathcal{U}}_{K}} \max _{\pi \in \Pi^{2}} E U\left(X_{T}^{t, x, y}(\pi, \nu), Y_{T}^{t, x, y}(\pi, \mu, \sigma)\right) .
\end{gathered}
$$

Therefore we find that the values of problems (2.1), (2.2) and (2.3), (2.4) are equal to

$$
\begin{aligned}
& \min _{\nu \in \tilde{\mathcal{U}}_{K}} \max _{\pi \in \Pi^{2}} E U\left(X_{T}^{t, x, y}(\pi, \nu), Y_{T}^{t, x, y}(\pi, \nu)\right) \\
& \quad=\max _{\pi \in \Pi^{2}} \min _{(\mu, \sigma) \in \mathcal{U}_{K}} E U\left(X_{T}^{t, x, y}(\pi, \mu, \sigma), Y_{T}^{t, x, y}(\pi, \mu, \sigma)\right) .
\end{aligned}
$$

Corollary 2.7 The optimal strategy of the robust utility maximization problem (2.1), (2.2) is given by

$$
\pi^{*}(t, x, y)=-\frac{(b(y)+\bar{\ell}(t, x, y)) v_{x}(t, x, y)+\bar{m}(t, x, y) \rho v_{x y}(t, x, y)}{\left(2 \sigma_{M} \bar{\ell}(t, x, y)-\sigma_{-} \sigma_{+}\right) v_{x x}(t, x, y)},
$$

where the pair $(\bar{\ell}(t, x, y), \bar{m}(t, x, y))$ is defined by (2.12) and $v(t, x, y)$ is a solution of (2.10), (2.11).

Example 2.8 Let us consider the robust mean-variance hedging problem with zero drift and unknown volatility

$$
\begin{aligned}
& \min _{\pi \in \Pi^{2}} \max _{\sigma_{t} \in\left[\sigma_{-}, \sigma_{+}\right]} E\left(H\left(Y_{T}\right)-X_{T}(\pi, \sigma)\right)^{2}, \\
& d X_{t}=r X_{t} d t+\pi_{t} \sigma_{t} d W_{t}, \quad X_{0}=x, \\
& d Y_{t}=\beta\left(Y_{t}\right) d t+\rho d W_{t}+\sqrt{1-\rho^{2}} d W_{t}^{\perp}, \quad Y_{0}=y .
\end{aligned}
$$

Therefore we have $U(x, y)=-(x-H(y))^{2},(x, y) \in \mathbb{R}^{2}, \mu_{-}=\mu_{+}=0, r^{\prime}(y)=0$. We assume that $H$ is a continuous bounded function. By (2.12), we get

$$
\left(p_{\mu} \cdot v^{*}(t, x, y), p_{\sigma} \cdot v^{*}(t, x, y)\right)=\left(0, \frac{\sigma_{-} \sigma_{+}}{\sigma_{M}}\right)
$$

because we have $p_{\sigma} \cdot v^{*}(t, x, y)=\frac{2 \sigma_{-} \sigma_{+}}{\sigma_{+}+\sigma_{-}}=\frac{\sigma_{-} \sigma_{+}}{\sigma_{M}}$. Note that this implies that $v^{*}(t, x, y)=\frac{\sigma_{-}}{\sigma_{+}+\sigma_{-}} \delta_{\left(0, \sigma_{+}\right)}+\frac{\sigma_{+}}{\sigma_{+}+\sigma_{-}} \delta_{\left(0, \sigma_{-}\right)}$. Thus

$$
\underset{\sigma \in\left[\sigma_{-}, \sigma_{+}\right]}{\operatorname{argmin}} \frac{\rho^{2} \sigma^{2}}{-2\left(2 \sigma_{M} \sigma-\sigma_{-} \sigma_{+}\right)}=\frac{\sigma_{-} \sigma_{+}}{\sigma_{M}}
$$

and it follows from (2.10) that 


$$
\begin{aligned}
& \frac{\partial}{\partial t} v(t, x, y)+\frac{1}{2} v_{y y}(t, x, y)+\beta(y) v_{y}(t, x, y)+x r(y) v_{x}(t, x, y) \\
& \quad+\min _{\sigma \in\left[\sigma_{-}, \sigma_{+}\right]} \frac{\rho^{2} \sigma^{2} v_{x y}^{2}(t, x, y)}{-2\left(2 \sigma_{M} \sigma-\sigma_{-} \sigma_{+}\right) v_{x x}(t, x, y)} \\
& =\frac{\partial}{\partial t} v(t, x, y)+\frac{1}{2} v_{y y}(t, x, y)+\beta(y) v_{y}(t, x, y)+x r(y) v_{x}(t, x, y) \\
& \quad-\rho^{2} \frac{\sigma_{-} \sigma_{+}}{2 \sigma_{M}^{2}} \frac{v_{x y}^{2}(t, x, y)}{v_{x x}(t, x, y)}=0 \\
& v(T, x, y)=-(x-H(y))^{2} .
\end{aligned}
$$

The solution of (2.15), (2.16) can be given as a quadratic polynomial in $x$,

$$
v(t, x, y)=-A(t, y) x^{2}+2 B(t, y) x-C(t, y),
$$

where the triplet $(A, B, C)$ satisfies the system of PDEs

$$
\begin{aligned}
& \frac{\partial}{\partial t} A(t, y)+\frac{1}{2} A_{y y}(t, y)+\beta(y) A_{y}(t, y)+2 r A(t, y)+\rho^{2} \frac{\sigma_{-} \sigma_{+}}{2 \sigma_{M}^{2}} \frac{A_{y}^{2}(t, y)}{A(t, y)}=0 \\
& A(T, y)=1 \\
& \frac{\partial}{\partial t} B(t, y)+\frac{1}{2} B_{y y}(t, y)+\beta(y) B_{y}(t, y)+2 r B(t, y) \\
& \quad+\rho^{2} \frac{\sigma_{-} \sigma_{+}}{2 \sigma_{M}^{2}} \frac{A_{y}(t, y) B_{y}(t, y)}{A(t, y)}=0 \\
& B(T, y)=H(y), \\
& \frac{\partial}{\partial t} C(t, y)+\frac{1}{2} C_{y y}(t, y)+\beta(y) C_{y}(t, y)+\rho^{2} \frac{\sigma_{-} \sigma_{+}}{2 \sigma_{M}^{2}} \frac{B_{y}^{2}(t, y)}{A(t, y)}=0 \\
& C(T, y)=H^{2}(y) .
\end{aligned}
$$

This system admits the explicit solution

$$
\begin{aligned}
& A(t, y)=e^{2 r(T-t)}, \\
& B(t, y)=e^{2 r(T-t)} E H\left(Y_{T}^{t, y}\right), \\
& C(t, y)=\rho^{2} \frac{\sigma_{-} \sigma_{+}}{2 \sigma_{M}^{2}} e^{2 r(T-t)} \int_{t}^{T} E B_{y}^{2}\left(s, Y_{s}^{t, x, y}\right) d s+E H^{2}\left(Y_{T}^{t, x, y}\right)
\end{aligned}
$$


(notice that $B_{y}(t, y)=e^{2 r(T-t)} E H_{y}\left(Y_{T}^{t, y}\right) e^{\int_{t}^{T} \beta_{y}\left(Y_{s}^{t, y}\right) d s}$, when $H$ is differentiable). The optimal strategy then takes the form

$$
\begin{aligned}
\pi^{*}(t, x, y) & =-\frac{\rho \frac{\sigma_{-} \sigma_{+}}{\sigma_{M}} v_{x y}(t, x, y)}{\left(2 \sigma_{M} \frac{\sigma_{-} \sigma_{+}}{\sigma_{M}}-\sigma_{-} \sigma_{+}\right) v_{x x}(t, x, y)} \\
& =-\frac{\rho}{\sigma_{M}} \frac{v_{x y}(t, x, y)}{v_{x x}(t, x, y)}=-\frac{\rho}{\sigma_{M}} \frac{B_{y}(t, y)-x A_{y}(t, y)}{-A(t, y)} \\
& =\frac{\rho}{\sigma_{M}} \frac{B_{y}(t, y)}{A(t, y)}=\frac{\rho}{\sigma_{M}} e^{-2 r(T-t)} B_{y}(t, y) .
\end{aligned}
$$

The function $B(t, y)=e^{2 r(T-t)} E H\left(Y_{T}^{t, y}\right)$ is a classical bounded solution of the corresponding linear parabolic equation with bounded continuous $B_{y}(t, y)$ and continuous $B_{y y}(t, y)$ (see [14], formulas (5.20)-(5.22) of Chap. VI). It is clear that

$$
|v(t, x, y)| \leq L\left(1+|x|^{2}\right), \quad\left|\frac{v_{x}(t, x, y)}{v_{x x}(t, x, y)}\right| \leq L(1+|x|), \quad\left|\frac{v_{x y}(t, x, y)}{v_{x x}(t, x, y)}\right| \leq L
$$

for some $L>0$ and $B_{y}(t, y)$ is a locally Lipschitz function. Hence the pair $\left(\pi^{*}(t, x, y), v^{*}(t, y)\right)$ satisfies all conditions of Theorem 2.6.

The case $\rho=1, r=0, \beta \equiv 0$ is discussed in the introduction. In this case the second equation in (2.4) defines a Brownian motion $Y=B$ for all non-anticipating strategies $v(Y)=v(B)$ and (2.4) coincides with (1.3).

In the case of an objective function $U(x, y)$ defined on $\mathbb{R}_{+} \times \mathbb{R}$, it is convenient to determine the wealth process as a solution of the SDE

$$
\begin{aligned}
d X_{t} & =r\left(Y_{t}\right) X_{t} d t+\pi_{t} X_{t}\left(b\left(Y_{t}\right)+\mu_{t}\right) d t+\pi_{t} X_{t} \sigma_{t} d W_{t}, \quad X_{0}=x, \\
d Y_{t} & =\beta\left(Y_{t}\right) d t+\rho d W_{t}+\sqrt{1-\rho^{2}} d W_{t}^{\perp}, \quad Y_{0}=y .
\end{aligned}
$$

The set of admissible strategies $\Pi$ is now defined as the set of all predictable processes $\pi$ such that $\int \pi_{s} d W_{s}$ is a BMO-martingale (as regards BMO-martingales, see [20]). It is clear that for each $(\pi, \mu, \sigma) \in \Pi \times \mathcal{U}_{K}, \int \pi_{s} \sigma_{s} d W_{s}$ is also a BMOmartingale, a solution of (2.17) is positive, and the maximin problem

$$
\max _{\pi \in \Pi} \min _{(\mu, \sigma) \in \mathcal{U}_{K}} E U\left(X_{T}^{\mu, \sigma}(\pi), Y_{T}\right),
$$

makes sense.

As in the previous case of the problem (2.1), (2.2), we consider the extended maximin problem

$$
\begin{gathered}
\max _{\pi \in \Pi} \min _{\nu \in \tilde{\mathcal{U}}_{K}} E U\left(X_{T}^{v}(\pi), Y_{T}^{v}\right) \\
d X_{t}^{v}=r\left(Y_{t}^{v}\right) X_{t}^{v} d t+\pi_{t} X_{t}^{v}\left(b\left(Y_{t}^{v}\right)+p_{\mu} \cdot v_{t}\right) d t+\pi_{t} X_{t}^{v} \sqrt{p_{\sigma}^{2} \cdot v_{t}} d W_{t}, \quad X_{0}^{v}=x,
\end{gathered}
$$




$$
d Y_{t}^{v}=\beta\left(Y_{t}^{v}\right) d t+\rho \frac{p_{\sigma} \cdot v_{t}}{\sqrt{p_{\sigma}^{2} \cdot v_{t}}} d W_{t}+\sqrt{1-\rho^{2} \frac{\left(p_{\sigma} \cdot v_{t}\right)^{2}}{p_{\sigma}^{2} \cdot v_{t}}} d W_{t}^{\perp}, \quad Y_{0}^{v}=y
$$

It is easy to see that the HJBI equation for the value $v(t, x, y)$ of this problem is same as (2.10), but $\pi^{*}$ is now defined by

$$
\pi^{*}(t, x, y)=-\frac{(b(y)+\bar{\ell}(t, x, y)) v_{x}(t, x, y)+\bar{m}(t, x, y) \rho v_{x y}(t, x, y)}{x\left(2 \sigma_{M} \bar{\ell}(t, x, y)-\sigma_{-} \sigma_{+}\right) v_{x x}(t, x, y)},
$$

where the pair $(\bar{\ell}(t, x, y), \bar{m}(t, x, y))$ is defined by (2.12).

Theorem 2.6' 1 Suppose that $v(t, x, y)$ is a classical solution of (2.7), (2.8) such that $v_{x x}(t, x, y)<0$ and such that for some constants $L>0, p \geq 1$ and for all $(t, x, y) \in[0, T] \times \mathbb{R}_{+} \times \mathbb{R}$,

$$
\begin{aligned}
|v(t, x, y)| & \leq L(1+|x|+|y|)^{p}, \\
\left|\frac{v_{x}(t, x, y)}{v_{x x}(t, x, y)}\right| & \leq L x, \\
\left|\frac{v_{x y}(t, x, y)}{v_{x x}(t, x, y)}\right| & \leq L x .
\end{aligned}
$$

Suppose also that the triplet $\left(\pi^{*}(t, x, y), p_{\mu} \cdot v^{*}(t, x, y), p_{\sigma} \cdot v^{*}(t, x, y)\right)$ satisfies a Lipschitz condition on each compact subset of $[0, T] \times \mathbb{R}_{+} \times \mathbb{R}$, where

$$
\begin{aligned}
\pi^{*}(t, x, y) & \\
=- & \frac{\left(b(y)+p_{\mu} \cdot v^{*}(t, x, y)\right) v_{x}(t, x, y)+p_{\sigma} \cdot v^{*}(t, x, y) \rho v_{x y}(t, x, y)}{x\left(2 \sigma_{M} p_{\sigma} \cdot v^{*}(t, x, y)-\sigma_{-} \sigma_{+}\right) v_{x x}(t, x, y)},
\end{aligned}
$$

and where $\left(p_{\mu} \cdot v^{*}(t, x, y), p_{\sigma} \cdot v^{*}(t, x, y)\right)$ coincides with $(\bar{\ell}(t, x, y), \bar{m}(t, x, y))$ defined by (2.12). Then $\left(\pi^{*}, v^{*}\right)$ is a saddle point of the problem (2.18), (2) and

$$
\begin{aligned}
\max _{\pi \in \Pi} \min _{(\mu, \sigma) \in \mathcal{U}_{K}} E U\left(X_{T}^{\mu, \sigma}(\pi), Y_{T}\right) & =\max _{\pi \in \Pi} \min _{\nu \in \tilde{\mathcal{U}}_{K}} E U\left(X_{T}^{v}(\pi), Y_{T}^{\nu}\right) \\
& =\min _{\nu \in \tilde{\mathcal{U}}_{K}} \max _{\pi \in \Pi} E U\left(X_{T}^{v}(\pi), Y_{T}^{v}\right) .
\end{aligned}
$$

Proof The strategy given by (2.20) is bounded as for $(t, x, y) \in[0, T] \times \mathbb{R}_{+} \times \mathbb{R}$,

$$
\left|\pi^{*}(t, x, y)\right| \leq \frac{\max _{y}\left(b(y)+\mu_{+}\right)}{\sigma_{-}^{2}}\left|\frac{v_{x}(t, x, y)}{x v_{x x}(t, x, y)}\right|+\frac{\rho \sigma_{+}}{\sigma_{-}^{2}}\left|\frac{v_{x y}(t, x, y)}{x v_{x x}(t, x, y)}\right| \leq \bar{L}
$$


for some constant $\bar{L}$. Hence $\left(\pi^{*}\left(t, X_{t}^{*}, Y_{t}^{*}\right)\right)_{t \in[0, T]} \in \Pi$, where $\left(X^{*}, Y^{*}\right)$ is the corresponding solution of (2). The rest of the proof follows the proof of Theorem 2.6.

\section{Power and exponential utility cases}

Now let us consider the robust utility maximization problem with the power utility $U(x)=\frac{1}{q} x^{q}, x>0$, with $0<q<1$,

$$
\max _{\pi \in \Pi} \min _{(\mu, \sigma) \in \mathcal{U}_{K}} \frac{1}{q} E\left(X_{T}^{\mu, \sigma}(\pi)\right)^{q},
$$

subject to

$$
\begin{aligned}
d X_{t} & =r\left(Y_{t}\right) X_{t} d t+\pi_{t} X_{t}\left(b\left(Y_{t}\right)+\mu_{t}\right) d t+\pi_{t} X_{t} \sigma_{t} d W_{t}, \quad X_{0}=x, \\
d Y_{t} & =\beta\left(Y_{t}\right) d t+\rho d W_{t}+\sqrt{1-\rho^{2}} d W_{t}^{\perp}, \quad Y_{0}=y .
\end{aligned}
$$

In this case, the HJBI equation (2.10), (2.11) becomes

$$
\begin{aligned}
& \frac{\partial}{\partial t} v(t, x, y)+\frac{1}{2} v_{y y}(t, x, y)+\beta(y) v_{y}(t, x, y)+x r(y) v_{x}(t, x, y) \\
& \quad+\min _{(\mu, \sigma) \in K} \frac{\left((b(y)+\mu) v_{x}(t, x, y)+\rho \sigma v_{x y}(t, x, y)\right)^{2}}{-2\left(2 \sigma_{M} \sigma-\sigma_{-} \sigma_{+}\right) v_{x x}(t, x, y)}=0, \\
& v(T, x, y)=\frac{1}{q} x^{q} .
\end{aligned}
$$

A solution of this equation is of the form $v(t, x, y)=\frac{1}{q} x^{q} e^{u(t, y)}$, where $u$ satisfies the equation

$$
\begin{aligned}
& \frac{\partial}{\partial t} u(t, y)+\frac{1}{2} u_{y y}(t, y)+\beta(y) u_{y}(t, y)+\frac{1}{2} u_{y}^{2}(t, y)+q r(y) \\
& -\frac{q}{2(q-1)} \min _{(\mu, \sigma) \in K} \frac{\left(b(y)+\mu+\rho \sigma u_{y}(t, y)\right)^{2}}{2 \sigma_{M} \sigma-\sigma_{-} \sigma_{+}}=0, \\
& u(T, y)=0 .
\end{aligned}
$$

The pair $\left(p_{\mu} \cdot v^{*}(t, x, y), p_{\sigma} \cdot v^{*}(t, x, y)\right)$ from Theorem 2.6 takes the form

$$
\left(p_{\mu} \cdot v^{*}(t, y), p_{\sigma} \cdot v^{*}(t, y)\right)=\left(\ell\left(\rho u_{y}(t, y)\right), m\left(\rho u_{y}(t, y)\right)\right),
$$

where $(\ell, m)$ is defined by (2.9). 
Remark 3.1 By Corollary 2.5 and (2.12), equation (3.3) can be written as

$$
\begin{aligned}
& \frac{\partial}{\partial t} u(t, y)+\frac{1}{2} u_{y y}(t, y)+\beta(y) u_{y}(t, y)+\frac{1}{2} u_{y}^{2}(t, y)+q r(y) \\
& \quad-\frac{q \rho u_{y}(t, y)}{2(q-1) \sigma_{M}^{2}}\left(2\left((y)+\mu_{+}\right) \sigma_{M}+\sigma_{-} \sigma_{+} \rho u_{y}(t, y)\right) \\
& \quad \times \chi\left(\rho u_{y}(t, y) \leq \frac{\mu_{+} \sigma_{M}}{\sigma_{M} \sigma_{-}-\sigma_{+} \sigma_{-}}\right) \\
& -\frac{q}{2(q-1) \sigma_{-}^{2}}\left(b(y)+\mu_{+}+\rho \sigma_{-} u_{y}(t, y)\right)^{2} \\
& \quad \times \chi\left(\frac{\mu_{+} \sigma_{M}}{\sigma_{M} \sigma_{-}-\sigma_{+} \sigma_{-}}<\rho u_{y}(t, y) \leq-\frac{\mu_{+}}{\sigma_{-}}\right) \\
& \quad-\frac{q}{2(q-1) \sigma_{+}^{2}}\left(b(y)+\mu_{-}+\rho \sigma_{+} u_{y}(t, y)\right)^{2} \\
& \quad \times \chi\left(-\frac{\mu_{-}}{\sigma_{+}}<\rho u_{y}(t, y) \leq \frac{\mu_{-} \sigma_{M}}{\sigma_{M} \sigma_{+}-\sigma_{+} \sigma_{-}}\right) \\
& \quad-\frac{q \rho u_{y}(t, y)}{2(q-1) \sigma_{M}^{2}}\left(2\left(b(y)+\mu_{-}\right) \sigma_{M}+\sigma_{-} \sigma_{+} \rho u_{y}(t, y)\right) \\
& \quad \times \chi\left(\rho u_{y}(t, y)>\frac{\mu}{\sigma_{M} \sigma_{+}-\sigma_{+} \sigma_{-}}\right)=0, \\
& u(T, y)=0 .
\end{aligned}
$$

Theorem 3.2 Under conditions (A1)-(A3), the Cauchy problem (3.3), (3.4) admits a classical solution with bounded $u_{y}(t, y)$, and a saddle point $\left(v^{*}(t, y), \pi^{*}(t, y)\right)$ of the problem (2.18), (2) is defined by equation (3.5) and by the formula

$$
\pi^{*}(t, y)=\frac{1}{1-q}\left(\frac{b(y)+p_{\mu} \cdot v^{*}(t, y)}{p_{\sigma}^{2} \cdot v^{*}(t, y)}+\rho \frac{p_{\sigma} \cdot v^{*}(t, y)}{p_{\sigma}^{2} \cdot v^{*}(t, y)} u_{y}(t, y)\right) .
$$

Moreover, $\pi^{*}(t, y)$ is the optimal strategy of the robust utility maximization problem (3.1), (3.2).

Proof By Proposition B.1 in Appendix B, there exists a classical solution of (3.3), (3.4) with bounded $u_{y}(t, y)$. From the continuity of $u_{y y}(t, y)$, it follows that $u_{y}(t, y)$ is a locally Lipschitz function. By Lemma A.2 below, the pair $\left(p_{\mu} \cdot v^{*}(t, y), p_{\sigma} \cdot v^{*}(t, y)\right)$, where $v^{*}(t, y)$ is defined by (3.5), is a locally Lipschitz function. Because we have $p_{\sigma}^{2} \cdot v^{*}(t, y)=2 \sigma_{M}\left(p_{\sigma} \cdot v^{*}(t, y)\right)-\sigma_{-} \sigma_{+} \geq \sigma_{-}^{2}$, also $\frac{1}{p_{\sigma}^{2} \cdot v^{*}(t, y)}$ is a locally Lipschitz function. Hence 


$$
\begin{aligned}
\pi^{*}(t, y) & =-\frac{1}{q-1} \frac{b(y)+\ell\left(\rho u_{y}(t, y)\right)+m\left(\rho u_{y}(t, y)\right) \rho u_{y}(t, y)}{2 m\left(\rho u_{y}(t, y)\right) \sigma_{M}-\sigma_{-} \sigma_{+}} \\
& =\frac{1}{1-q} \frac{b(y)+p_{\mu} \cdot v^{*}(t, y)+p_{\sigma} \cdot v^{*}(t, y) \rho u_{y}(t, y)}{2 p_{\sigma} \cdot v^{*}(t, y) \sigma_{M}-\sigma_{-} \sigma_{+}} \\
& =\frac{1}{1-q}\left(\frac{b(y)+p_{\mu} \cdot v^{*}(t, y)}{p_{\sigma}^{2} \cdot v^{*}(t, y)}+\rho \frac{p_{\sigma} \cdot v^{*}(t, y)}{p_{\sigma}^{2} \cdot v^{*}(t, y)} u_{y}(t, y)\right)
\end{aligned}
$$

from (2.14) is also a Lipschitz function. It is obvious that we have $\pi^{*} \in \Pi$ for each $v \in \mathcal{U}_{K}$ (since $X\left(\pi^{*}, v\right)$ is a solution of a linear SDE); moreover, we have $v_{x x}(t, x, y)=(q-1) x^{q-2} e^{u(t, y)}<0$ and all the conditions of Theorem 2.6' are satisfied. Therefore we can conclude that $\left(\pi^{*}(t, y), v^{*}(t, y)\right)$ is a saddle point of the problem (3.1), (2.5).

Corollary 3.3 If $b=0, r=0$, then

$$
u(t, y)=-\frac{q}{2(q-1)}(T-t) \min _{(\mu, \sigma) \in K} \frac{\mu^{2}}{2 \sigma_{M} \sigma-\sigma_{-} \sigma_{+}}=-\frac{q}{2(q-1)}(T-t) \frac{\mu_{-}^{2}}{\sigma_{+}^{2}}
$$

is a solution of (3.3), and a saddle point of the maximin problem can be given explicitly as

$$
\left(\mu_{t}^{*}, \sigma_{t}^{*}\right)=\left(\mu_{-}, \sigma_{+}\right), \quad \pi^{*}(t, x, y)=-\frac{\mu_{-}}{2(q-1) \sigma_{+}^{2}} x .
$$

Example 3.4 When $\sigma_{-}=\sigma_{+}=\sigma_{M}$, we obtain

$$
\begin{aligned}
& \frac{\partial}{\partial t} u(t, y)+\frac{1}{2} u_{y y}(t, y)+\beta(y) u_{y}(t, y)+\frac{1}{2} u_{y}^{2}(t, y) \\
& \quad-\frac{q}{2(q-1) \sigma_{M}^{2}} \min _{\mu-\leq \leq \mu_{+}}\left(b(y)+\mu+\rho \sigma_{M} u_{y}(t, y)\right)^{2} \\
& =\frac{\partial}{\partial t} u(t, y)+\frac{1}{2} u_{y y}(t, y)+\left(2 \rho \sigma_{M} b(y)+\beta(y)\right) u_{y}(t, y) \\
& \quad+\frac{1}{2}\left(1-\frac{q \rho^{2} \sigma_{M}}{q-1}\right) u_{y}^{2}(t, y) \\
& \quad-\frac{q}{2(q-1) \sigma_{M}^{2}} \min _{-\leq \mu \leq \mu_{+}}\left((b(y)+\mu)^{2}+2 \mu \rho \sigma_{M} u_{y}(t, y)\right)=0, \\
& u(T, y)=0
\end{aligned}
$$

Applications of such equations in finance and the existence of a classical solution are discussed in [16]. 
Remark 3.5 Instead of the PDE (3.3), we can use the BSDE with quadratic growth

$$
\begin{aligned}
d V_{t}= & -\left(\frac{1}{2} Z_{t}^{2}+q r\left(Y_{t}\right)-\frac{q}{2(q-1)} \min _{(\mu, \sigma) \in K} \frac{\left(b\left(Y_{t}\right)+\mu+\rho \sigma Z_{t}\right)^{2}}{2 \sigma_{M} \sigma-\sigma_{-} \sigma_{+}}\right) d t \\
& +Z_{t} d W_{t}+Z_{t}^{\perp} d W_{t}^{\perp}, \\
V_{T}= & 0,
\end{aligned}
$$

whose solvability follows from the results of [21,33]. The solution of the BSDE can be constructed, using the solution of the PDE (3.3), by the formulas

$$
V_{t}=u\left(t, Y_{t}\right), \quad Z_{t}=\rho u_{y}\left(t, Y_{t}\right), \quad Z_{t}^{\perp}=\sqrt{1-\rho^{2}} u_{y}\left(t, Y_{t}\right) .
$$

The optimal strategy $\pi_{t}^{*}=\pi^{*}\left(t, Y_{t}\right)$ is defined by the linear equation

$$
\pi_{t}^{*}=\frac{1}{1-q}\left(\frac{b\left(Y_{t}\right)+p_{\mu} \cdot \hat{v}_{t}^{*}\left(Z_{t}\right)}{p_{\sigma}^{2} \cdot \hat{v}_{t}^{*}\left(Z_{t}\right)}+\frac{p_{\sigma} \cdot \hat{v}_{t}^{*}\left(Z_{t}\right)}{p_{\sigma}^{2} \cdot \hat{v}_{t}^{*}\left(Z_{t}\right)} Z_{t}\right),
$$

following from (3.6). As follows from (3.5), the pair $\left(p_{\mu} \cdot \hat{v}_{t}^{*}(z), p_{\sigma} \cdot \hat{v}_{t}^{*}(z)\right)$ coincides with $(\ell(z), m(z))$ defined by (2.9).

Suppose now $U(x, y)=-e^{-\gamma(x-H(y))},(x, y) \in \mathbb{R}^{2}, \gamma>0$ and $r=0$. This case corresponds to the exponential hedging problem of the contingent claim $H(y)$, depending only on the non-tradable asset. We assume that $H \in C_{b}(\mathbb{R})$. Now following [26], we consider the restricted class of strategies $\Pi=\left\{\pi \in \Pi^{2}: \int_{0}^{t} \pi_{s} d W_{s}\right.$ is a BMO-martingale and the minimax problem

$$
\min _{\pi \in \Pi} \max _{(\mu, \sigma) \in \mathcal{U}_{K}} E e^{\gamma\left(H\left(Y_{T}\right)-X_{T}^{\mu, \sigma}(\pi)\right)}
$$

subject to (2.2). It is easy to verify that a solution of (2.10), (2.11) is of the form $v(t, x, y)=-e^{\gamma u(t, y)-\gamma x}$, where $u(t, y)$ is a bounded solution of

$$
\begin{aligned}
& \frac{\partial}{\partial t} u(t, y)+\frac{1}{2} u_{y y}(t, y)+\beta(y) u_{y}(t, y)+\frac{1}{2} \gamma u_{y}^{2}(t, y) \\
& +\frac{1}{2 \gamma} \min _{(\mu, \sigma) \in K} \frac{\left(b(y)+\mu+\rho \gamma \sigma u_{y}(t, y)\right)^{2}}{2 \sigma_{M} \sigma-\sigma_{-} \sigma_{+}}=0, \\
& u(T, y)=H(y) .
\end{aligned}
$$

The existence of a classical bounded solution of (3.8), (3.9) with bounded $u_{y}$ for the case

$$
H^{\prime} \in C_{0}(\mathbb{R})
$$

follows from Proposition B.1. Thus $\frac{v_{x}(t, x, y)}{v_{x x}(t, x, y)}=-\frac{1}{\gamma}, \frac{v_{x y}(t, x, y)}{v_{x x}(t, x, y)}=-u_{y}(t, y)$ are bounded. One can check that all conditions of Theorem 2.6 except for the polynomial growth condition of $v(t, x, y)$ are satisfied. The robust optimal portfolio is 


$$
\pi^{*}(t, y)=-\frac{1}{\gamma} \frac{b(y)+p_{\mu} \cdot v^{*}(t, y)-\gamma \rho p_{\sigma} \cdot v^{*}(t, y) u_{y}(t, y)}{2 \sigma_{M} p_{\sigma} \cdot v^{*}(t, y)-\sigma_{-} \sigma_{+}},
$$

with $\left(p_{\mu} \cdot v^{*}(t, y), p_{\sigma} \cdot v^{*}(t, y)\right)$ from (2.12). So $\left(\pi^{*}(t, y), p_{\mu} \cdot v^{*}(t, y), p_{\sigma} \cdot v^{*}(t, y)\right)$ is a bounded, locally Lipschitz function of $(t, y)$ and $X_{s}^{t, x, y}\left(\pi^{*}, v^{*}\right), s \geq t$, is a BMOmartingale. Hence $\left\{e^{\gamma\left(X_{\tau}^{t, x, y}\left(\pi^{*}, v^{*}\right)-u\left(\tau, Y_{\tau}^{t, x, y}\right)\right)}: \tau\right.$ stopping time, $\left.t \leq \tau \leq T\right\}$ is a uniformly integrable family of random variables. This enables us to pass to the limit in Theorem 2.6 and obtain

$$
\begin{aligned}
& E v\left(X_{T}^{* t, x, y}, Y_{T}^{* t, x, y}\right)=E e^{\gamma\left(X_{\tau_{R}}^{* t, x, y}-u\left(\tau_{R}, Y_{\tau_{R}}^{* t, x, y}\right)\right)} \\
& \quad \longrightarrow \quad E e^{\gamma\left(X_{T}^{* t, x, y}-u\left(T, Y_{T}^{* t, x, y}\right)\right)}=E v\left(T, X_{T}^{* t, x, y}, Y_{T}^{* t, x, y}\right) \quad \text { as } R \rightarrow \infty
\end{aligned}
$$

without the polynomial growth condition of $v(t, x, y)$. Hence we have proved

Theorem 3.6 Under conditions (A1)-(A3) and (3.10), the Cauchy problem (3.8), (3.9) admits a classical solution with bounded $u_{y}(t, y)$, and a saddle point $\left(\pi^{*}(t, y)\right.$, $\left.v^{*}(t, y)\right)$ of the problem (3.7) is defined by the equation

$$
\left(p_{\mu} \cdot v^{*}(t, y), p_{\sigma} \cdot v^{*}(t, y)\right)=\left(\ell\left(\rho \gamma u_{y}(t, y)\right), m\left(\rho \gamma u_{y}(t, y)\right)\right)
$$

and by the formula

$$
\pi^{*}(t, y)=-\frac{1}{\gamma} \frac{b(y)+p_{\mu} \cdot v^{*}(t, y)-\gamma \rho\left(p_{\sigma} \cdot v^{*}(t, y)\right) u_{y}(t, y)}{2 \sigma_{M} p_{\sigma} \cdot v^{*}(t, y)-\sigma_{-} \sigma_{+}} .
$$

Moreover, $\pi^{*}(t, y)$ is an optimal strategy for the robust exponential hedging problem (3.7), (2.2).

Acknowledgements The authors are grateful to the co-editor, the associate editor and an anonymous referee for careful reading and helpful suggestions for the revision of this paper. This study was supported by Georgian National Science Foundation grant GNSF/ST09-471-3-104.

Open Access This article is distributed under the terms of the Creative Commons Attribution License which permits any use, distribution, and reproduction in any medium, provided the original author(s) and the source are credited.

\section{Appendix A}

Each measure $v$ may be realized as the distribution of a pair of random variables $(\xi, \eta)$ with values in $K$. Simplifying the notation, we denote $b(y)+\mu$ by $\mu$ again. Our aim is to characterize the dependence of the minimizer for the problem

$$
\min _{v \in \mathcal{P}(K)} \frac{\left(p_{\mu} \cdot v+\kappa p_{\sigma} \cdot v\right)^{2}}{p_{\sigma}^{2} \cdot v}=\min _{(\xi, \eta) \in K} \frac{(E \xi+\kappa E \eta)^{2}}{E \eta^{2}}
$$

on a parameter $\kappa \in \mathbb{R}$. 


\section{Proposition A.1 Let}

$$
\left(\xi^{*}, \eta^{*}\right)=\underset{(\xi, \eta) \in K}{\operatorname{argmin}} \frac{(E \xi+\kappa E \eta)^{2}}{E \eta^{2}} .
$$

Then $\xi^{*}$ is a number, $\eta^{*}$ is a Bernoulli random variable with values in the set $\left\{\sigma_{-}, \sigma_{+}\right\}$, and the expectation of the pair $\left(\xi^{*}, \eta^{*}\right)$ is given by the formula

$$
\left(\xi^{*}, E \eta^{*}\right)= \begin{cases}\left(\mu_{+}, \frac{\mu_{+}}{\kappa}+\frac{\sigma_{-} \sigma_{+}}{\sigma_{M}}\right) & \text { if } \kappa \in\left(-\infty, \frac{\mu_{+} \sigma_{M}}{\sigma_{M} \sigma_{-}-\sigma_{+} \sigma_{-}}\right], \\ \left(\mu_{+}, \sigma_{-}\right) & \text {if } \kappa \in\left(\frac{\mu_{+} \sigma_{M}}{\sigma_{M} \sigma_{-}-\sigma_{+} \sigma_{-}},-\frac{\mu_{+}}{\sigma_{-}}\right], \\ (\kappa,-1) \text { constant } & \text { if } \kappa \in\left(-\frac{\mu_{+}}{\sigma_{-}},-\frac{\mu_{-}}{\sigma_{+}}\right], \\ \left(\mu_{-}, \sigma_{+}\right) & \text {if } \kappa \in\left(-\frac{\mu_{-}}{\sigma_{+}}, \frac{\mu_{-} \sigma_{M}}{\sigma_{M} \sigma_{+}-\sigma_{+} \sigma_{-}}\right], \\ \left(\mu_{-}, \frac{\mu_{-}}{\kappa}+\frac{\sigma_{-} \sigma_{+}}{\sigma_{M}}\right) & \text { if } \kappa \in\left(\frac{\mu_{-} \sigma_{M}}{\sigma_{M} \sigma_{+}-\sigma_{+} \sigma_{-}}, \infty\right) .\end{cases}
$$

Moreover,

$$
\frac{\left(\xi^{*}+\kappa E \eta^{*}\right)^{2}}{E \eta^{* 2}}= \begin{cases}\frac{\kappa\left(2 \mu_{+} \sigma_{M}+\kappa \sigma_{-} \sigma_{+}\right)}{\sigma_{M}^{2}} & \text { if } \kappa \in\left(-\infty, \frac{\mu_{+} \sigma_{M}}{\sigma_{M} \sigma_{-}-\sigma_{+} \sigma_{-}}\right], \\ \frac{\left(\mu_{+}+\kappa \sigma_{-}\right)^{2}}{\sigma_{-}^{2}} & \text { if } \kappa \in\left(\frac{\mu_{+} \sigma_{M}}{\sigma_{M} \sigma_{-}-\sigma_{+} \sigma_{-}},-\frac{\mu_{+}}{\sigma_{-}}\right], \\ 0 & \text { if } \kappa \in\left(-\frac{\mu_{+}}{\sigma_{-}},-\frac{\mu_{-}}{\sigma_{+}}\right], \\ \frac{\left(\mu_{-}+\kappa \sigma_{+}\right)^{2}}{\sigma_{+}^{2}} & \text { if } \kappa \in\left(-\frac{\mu_{-}}{\sigma_{+}}, \frac{\mu_{-} \sigma_{M}}{\sigma_{M} \sigma_{+}-\sigma_{+} \sigma_{-}}\right], \\ \frac{\kappa\left(2 \mu-\sigma_{M}+\kappa \sigma_{-} \sigma_{+}\right)}{\sigma_{M}^{2}} & \text { if } \kappa \in\left(\frac{\mu_{-} \sigma_{M}}{\sigma_{M} \sigma_{+}-\sigma_{+} \sigma_{-}}, \infty\right) .\end{cases}
$$

Proof Let $\left(\mu_{+}+\kappa \sigma_{-}\right)\left(\mu_{-}+\kappa \sigma_{+}\right) \leq 0$. Then by the continuity of the function $\mu+\kappa \sigma,(\mu, \sigma) \in K$, there exists $(\hat{\mu}, \hat{\sigma})$ such that $\hat{\mu}+\kappa \hat{\sigma}=0$. Thus $(\hat{\mu}, \hat{\sigma})$ is proportional to $(\kappa,-1)$ and $\frac{\left(E \xi^{*}+\kappa E \eta^{*}\right)^{2}}{E \eta^{* 2}}=0$. If $\left(\mu_{+}+\kappa \sigma_{-}\right)\left(\mu_{-}+\kappa \sigma_{+}\right)>0$, then either $\kappa>\frac{\mu_{-}}{\sigma_{+}}$and $\xi^{*}=\mu_{-}$or $\kappa<-\frac{\mu_{+}}{\sigma_{-}}$and $\xi^{*}=\mu_{+}$. Thus it is sufficient to study the minimization problem

$$
\min _{\eta \in\left[\sigma_{-}, \sigma_{+}\right]} \frac{\left(\mu_{a}+\kappa E \eta\right)^{2}}{E \eta^{2}} \text { for } a=+,-.
$$

Now we show that $\eta^{*}$ is of the form $\eta^{*}=\sigma_{-} \chi_{B}+\sigma_{+} \chi_{B^{c}}$ for some event $B$. Indeed, if $E \eta^{*}=y$, then $E \eta^{* 2}=2 \sigma_{M} y-\sigma_{-} \sigma_{+}$and $\eta^{*}$ is the maximizer of the problem

$$
\max _{\eta, E \eta=y} E \eta^{2}
$$

since for any $\eta$ with $E \eta=y$, we have

$$
\begin{aligned}
E \eta^{2} & =E\left(\eta-\sigma_{M}\right)^{2}+2 \sigma_{M} y-\sigma_{M}^{2} \\
& \leq\left(\frac{\sigma_{+}-\sigma_{-}}{2}\right)^{2}+2 \sigma_{M} y-\sigma_{M}^{2}=2 \sigma_{M} y-\sigma_{-} \sigma_{+}=E \eta^{* 2} .
\end{aligned}
$$


Hence

$$
\min _{\eta \in\left[\sigma_{-}, \sigma_{+}\right]} \frac{\left(\mu_{a}+\kappa E \eta\right)^{2}}{E \eta^{2}}=\min _{\sigma_{-} \leq y \leq \sigma_{+}} \psi_{a}(y),
$$

where $\psi_{a}(y)=\frac{\left(\mu_{a}+\kappa y\right)^{2}}{2 \sigma_{M} y-\sigma_{-} \sigma_{+}}$. Since

$$
\psi_{a}^{\prime}(y)=\frac{\kappa^{2}}{2 \sigma_{M}}-\frac{\kappa^{2}}{2 \sigma_{M}} \frac{\left(2 \sigma_{M} \frac{\mu_{a}}{\kappa}+\sigma_{-} \sigma_{+}\right)^{2}}{\left(2 \sigma_{M} y-\sigma_{-} \sigma_{+}\right)^{2}},
$$

the equation $\psi_{a}^{\prime}(y)=0$ has the two roots

$$
y_{1}^{a}=-\frac{\mu_{a}}{\kappa}, \quad y_{2}^{a}=\frac{\mu_{a}}{\kappa}+\frac{\sigma_{-} \sigma_{+}}{\sigma_{M}} .
$$

If $y_{1}^{a}=-\frac{\mu_{a}}{\kappa} \in\left[\sigma_{-}, \sigma_{+}\right]$, then $y_{2}^{a}=\frac{\mu_{a}}{\kappa}+\frac{\sigma_{-} \sigma_{+}}{\sigma_{M}} \in\left[-\sigma_{+}+\frac{\sigma_{-} \sigma_{+}}{\sigma_{M}},-\sigma_{-}+\frac{\sigma_{-} \sigma_{+}}{\sigma_{M}}\right]$ and vice versa. Moreover, $\left[\sigma_{-}, \sigma_{+}\right] \cap\left[-\sigma_{+}+\frac{\sigma_{-} \sigma_{+}}{\sigma_{M}},-\sigma_{-}+\frac{\sigma_{-} \sigma_{+}}{\sigma_{M}}\right]=\emptyset$. Since $\lim _{y \rightarrow \pm \infty} \psi_{a}(y)= \pm \infty$, the smaller root is the maximizer and the bigger one the minimizer. The case of $y_{1}^{a} \in\left[\sigma_{-}, \sigma_{+}\right]$is equivalent to the case

$$
\kappa \in\left[-\frac{\sigma_{+}}{\mu_{a}},-\frac{\sigma_{-}}{\mu_{a}}\right],
$$

which yields $\min \psi_{a}(y)=\psi_{a}\left(y_{1}^{a}\right)=0$. From the relation $y_{2}^{a} \in\left[\sigma_{-}, \sigma_{+}\right]$, it follows that $-\sigma_{+}+\frac{\sigma_{-} \sigma_{+}}{\sigma_{M}} \leq-\frac{\mu_{a}}{\kappa} \leq-\sigma_{+}-\frac{\sigma_{-} \sigma_{+}}{\sigma_{M}}$, which is equivalent to the relation

$$
\kappa \in\left(-\infty, \frac{\mu_{a}}{\sigma_{-}-\frac{\sigma_{-} \sigma_{+}}{\sigma_{M}}}\right] \cup\left[\frac{\mu_{a}}{\sigma_{+}-\frac{\sigma_{-} \sigma_{+}}{\sigma_{M}}}, \infty\right) .
$$

In that case, $\min _{\sigma_{-} \leq y \leq \sigma_{+}} \psi_{a}(y)=\psi_{a}\left(y_{2}^{a}\right)=\kappa \frac{2 \mu_{a}+\kappa \sigma_{-} \sigma_{+}}{\sigma_{M}^{2}}$.

Now we consider step by step all the possibilities of displacement of $\kappa$ in the intervals formulated in the proposition.

(1) $\kappa \in\left(-\infty, \frac{\mu_{a}}{\sigma_{-}-\frac{\sigma_{-} \sigma_{+}}{\sigma_{M}}}\right]$ : Since $\frac{\mu_{a}}{\sigma_{-}-\frac{\sigma_{-} \sigma_{+}}{\sigma_{M}}} \leq-\frac{\mu_{+}}{\sigma_{-}}$, we have $\kappa \in\left(-\infty,-\frac{\mu_{+}}{\sigma_{-}}\right]$and $\xi^{*}=\mu_{+}$. Moreover, $\min \psi_{+}(y)=\psi_{+}\left(y_{2}^{+}\right)=\kappa \frac{2 \mu_{+}+\kappa \sigma_{-} \sigma_{+}}{\sigma_{M}^{2}}$.

(2) $\kappa \in\left(\frac{\mu_{+}}{\sigma_{-}-\frac{\sigma_{-} \sigma_{+}}{\sigma_{M}}},-\frac{\mu_{+}}{\sigma_{-}}\right]$: From $\kappa \leq-\frac{\mu_{+}}{\sigma_{-}}$, it follows that $y_{1}^{+}=-\frac{\mu_{+}}{\kappa}<\sigma_{-}$, and from $\kappa>\frac{\mu_{+}}{\sigma_{-}-\frac{\sigma_{-} \sigma_{+}}{\sigma_{M}}}$, it follows that $y_{2}^{+}=\frac{\mu_{+}}{\kappa}+\frac{\sigma_{-} \sigma_{+}}{\sigma_{M}}<\sigma_{-}$. Hence $\psi_{+}(y)$ is increasing on $\left[\sigma_{-}, \sigma_{+}\right]$and arg $\min _{\sigma_{-} \leq y \leq \sigma_{+}} \psi_{+}(y)=\sigma_{-}$.

(3) $\kappa \in\left(-\frac{\mu_{+}}{\sigma_{-}},-\frac{\mu_{-}}{\sigma_{+}}\right]$: Then $y_{1}^{+}=-\frac{\mu_{+}}{\kappa} \in\left[\sigma_{-}, \sigma_{+}\right]$and $\min \psi_{+}(y)=0$.

(4) $\kappa \in\left(-\frac{\mu_{-}}{\sigma_{+}}, \frac{\mu_{-}}{\sigma_{+}-\frac{\sigma_{-} \sigma_{+}}{\sigma_{M}}}\right]$ : In this case, we obtain $\frac{\mu_{-}}{\kappa}>\sigma_{+}-\frac{\sigma_{-} \sigma_{+}}{\sigma_{M}}$ and $y_{1}^{-}=-\frac{\mu_{-}}{\kappa}<-\sigma_{+}+\frac{\sigma_{-} \sigma_{+}}{\sigma_{M}}<\sigma_{-}, y_{2}^{-}=\frac{\mu_{-}}{\kappa}+\frac{\sigma_{-} \sigma_{+}}{\sigma_{M}}>\sigma_{+}$. Therefore $\psi_{-}(y)$ is decreasing on $\left[\sigma_{-}, \sigma_{+}\right]$and $\arg \min \psi_{+}(y)=\sigma_{+}$.

(5) $\kappa \in\left(\frac{\mu_{-}}{\sigma_{+}-\frac{\sigma_{-} \sigma_{+}}{\sigma_{M}}}, \infty\right]$ : Then $\kappa>\frac{\mu_{-}}{\sigma_{+}}$and $\xi^{*}=\mu_{-}$. On the other hand, from $\frac{\mu_{-}}{\kappa}<\sigma_{+}-\frac{\sigma_{-} \sigma_{+}}{\sigma_{M}}$, it follows that $y_{2}^{-} \in\left[\sigma_{-}, \sigma_{+}\right]$. So we obtain $\min _{\sigma_{-} \leq y \leq \sigma_{+}} \psi_{-}(y)=\psi_{-}\left(y_{2}^{-}\right)$. 
Lemma A.2 Suppose that $u(y), f_{1}(z), f_{2}(z), \ldots, f_{N}(z)$ are Lipschitz functions, and let $-\infty=a_{0}<a_{1}<\cdots<a_{N}=\infty$ be points satisfying that $f_{k}\left(a_{k}\right)=f_{k+1}\left(a_{k}\right)$, $k=1, \ldots, N-1$. Then the function

$$
v(y)=f_{k}(u(y)) \quad \text { if } a_{k-1}<u(y) \leq a_{k}, k=2, \ldots, N,
$$

is also a Lipschitz function.

Proof For the sake of simplicity we consider the case $N=2$. It is clear that $f_{k}(u(y)), k=1,2,3$, are Lipschitz functions, i.e., there exists a constant $C>0$ such that $\mid f_{k}\left(u\left(y_{1}\right)-f_{k}\left(u\left(y_{2}\right)\right)|\leq C| y_{1}-y_{2} \mid\right.$. Suppose that $A_{1}=\left\{y: u(y) \leq a_{1}\right\}$, $A_{2}=\left\{y: u(y)>a_{1}\right\}$ and take $y_{1} \in A_{1}, y_{2} \in A_{2}$. Since $u\left(y_{1}\right) \leq a_{1} \leq u\left(y_{2}\right)$, by the continuity of $u$ there exists $\bar{y}$ such that $u(\bar{y})=a_{1}, y_{1} \leq \bar{y} \leq y_{2}$. Hence we have

$$
\begin{aligned}
\left|v\left(y_{1}\right)-v\left(y_{2}\right)\right| & =\left|f_{1}\left(u\left(y_{1}\right)\right)-f_{2}\left(u\left(y_{2}\right)\right)\right| \\
& =\left|f_{1}\left(u\left(y_{1}\right)\right)-f_{1}\left(a_{1}\right)+f_{2}\left(a_{2}\right)-f_{2}\left(u\left(y_{2}\right)\right)\right| \\
& \leq\left|f_{1}\left(u\left(y_{1}\right)\right)-f_{1}(u(\bar{y}))\right|+\left|f_{2}(u(\bar{y}))-f_{2}\left(u\left(y_{2}\right)\right)\right| \\
& \leq C\left|y_{1}-\bar{y}\right|+C\left|y_{2}-\bar{y}\right|=C\left(y_{2}-y_{1}\right) .
\end{aligned}
$$

\section{Appendix B}

Let $\beta, a, b, H \in C_{b}(\mathbb{R})$ and $\gamma, c, g$ be some constants. We consider the Cauchy problem

$$
\begin{aligned}
& \frac{\partial}{\partial t} u(t, y)+\frac{1}{2} u_{y y}(t, y)+\beta(y) u_{y}(t, y)+\gamma u_{y}^{2}(t, y)+a(y) \\
& \quad+c \min _{(\mu, \sigma) \in K} \frac{\left(b(y)+\mu+g \sigma u_{y}(t, y)\right)^{2}}{2 \sigma_{M} \sigma-\sigma_{-} \sigma_{+}}=0, \\
& u(T, y)=H(y) .
\end{aligned}
$$

Proposition B.1 Let $\beta, a, b, H$ be such that $a^{\prime}, b^{\prime}, H^{\prime} \in C_{0}(\mathbb{R})$. Then the Cauchy problem (B.1), (B.2) admits a classical solution with bounded $u(t, y), u_{y}(t, y)$.

Proof By assumption, there exists $N \geq 0$ with $a^{\prime}(y), b^{\prime}(y)=0$ and $H^{\prime}(y)=0$ if $|y|>N$. Thus $a(y)=a^{+}, b(y)=b^{+}, H(y)=H^{+}$if $y \geq N$ and $a(y)=a^{-}$, $b(y)=b^{-}, H(y)=H^{-}$if $y \leq-N$, for some constants $a^{+}, a^{-}, b^{+}, b^{-}, H^{+}, H^{-}$. The solutions of (3.3) on the intervals $(-\infty,-N]$ and $[N, \infty)$ are 


$$
\begin{aligned}
& u^{-}(t)=a^{-}(T-t)+c \frac{\left(b^{-}+\mu_{-}\right)^{2}}{\sigma_{+}^{2}}(T-t)+H^{-}, \\
& u^{+}(t)=a^{+}(T-t)+c \frac{\left(b^{+}+\mu_{-}\right)^{2}}{\sigma_{+}^{2}}(T-t)+H^{+},
\end{aligned}
$$

respectively. Now let us consider on the bounded domain $(0, T) \times(-N, N)$ the Cauchy-Dirichlet problem

$$
\begin{aligned}
& \frac{\partial}{\partial t} u(t, y)+\frac{1}{2} u_{y y}(t, y)+\beta(y) u_{y}(t, y)+\gamma u_{y}^{2}(t, y)+d(y) \\
& \quad+c \min _{(\mu, \sigma) \in K} \frac{\left(b(y)+\mu+g \sigma u_{y}(t, y)\right)^{2}}{2 \sigma_{M} \sigma-\sigma_{-} \sigma_{+}}=0, \\
& u(T, y)=H(y), u(t, \pm N)=u^{ \pm}(t) .
\end{aligned}
$$

Suppose

$$
\begin{aligned}
& a_{1}(t, y, u, p)=\frac{1}{2} p \\
& a(t, y, u, p)=-\beta(y) p-\gamma p^{2}-d(y)-c \min _{(\mu, \sigma) \in K} \frac{(b(y)+\mu+g \sigma p)^{2}}{2 \sigma_{M} \sigma-\sigma_{-} \sigma_{+}} .
\end{aligned}
$$

Hence we get the Cauchy-Dirichlet problem for $\tilde{u}(t, y)=u(T-t, y)$ in the form of [23] as

$$
\begin{gathered}
\frac{\partial}{\partial t} \tilde{u}(t, y)-\frac{\partial}{\partial y} a_{1}\left(t, y, \tilde{u}(t, y), \tilde{u}_{y}(t, y)\right)+a\left(t, y, \tilde{u}(t, y), \tilde{u}_{y}(t, y)\right)=0, \\
\tilde{u}(0, y)=H(y), \tilde{u}(t, \pm N)=u^{ \pm}(T-t) .
\end{gathered}
$$

It is easy to see that $a(t, y, u, p)$ is a Lipschitz function on each ball of its domain, $a(t, y, u, 0) u$ is lower-bounded by a quadratic function of the type $-b_{1} u^{2}-b_{2}$, $b_{1}, b_{2}>0$, and all the other conditions of Theorem V.6.2 of [23] are satisfied. Therefore there exists a classical solution of (3.3), (3.4) with bounded $u_{y}(t, y)$.

Remark B.2 The existence of a classical solution to (B.3) with boundary conditions $u(T, y)=0, u_{y}(t, \pm N)+u(t, \pm N)=u^{ \pm}(t)$ follows also from Example 3.6 of [18].

\section{References}

1. Aubin, J.-P., Ekeland, I.: Applied Nonlinear Analysis. Wiley, New York (1984)

2. Avellaneda, M., Levy, A., Parás, A.: Pricing and hedging derivative securities in markets with uncertain volatility. Appl. Math. Finance 2, 73-88 (1995)

3. Ahn, H., Muni, A., Swindle, G.: Misspecified asset price models and robust hedging strategies. Appl. Math. Finance 4, 21-36 (1997)

4. Ahn, H., Muni, A., Swindle, G.: Optimal hedging strategies for misspecified asset price models. Appl. Math. Finance 6, 197-208 (1999) 
5. Bordigoni, G., Matoussi, A., Schweizer, M.: A stochastic control approach to a robust utility maximization problem. In: Benth, F.E., et al. (eds.) Stochastic Analysis and Applications. Proceedings of the Second Abel Symposium, Oslo, pp. 125-151. Springer, Berlin (2007)

6. Buff, R.: Uncertain Volatility Models-Theory and Applications. Springer, Berlin (2002)

7. Chen, Z., Epstein, L.: Ambiguity, risk, and asset returns in continuous time. Econometrica 70, 1403$1443(2002)$

8. Cheridito, P., Soner, H.M., Touzi, N., Victoir, N.: Second order backward stochastic differential equations and fully non-linear parabolic PDEs. Commun. Pure Appl. Math. 60, 1081-1110 (2007)

9. Cvitanić, J., Karatzas, I.: On dynamic measures of risk. Finance Stoch. 3, 451-482 (1999)

10. Denis, L., Kervarec, M.: Utility functions and optimal investment in non-dominated models (2009). http://hal.archives-ouvertes.fr/hal-00371215/en/

11. Denis, L., Martini, C.: A theoretical framework for the pricing of contingent claims in the presence of model uncertainty. Ann. Appl. Probab. 16, 827-852 (2006)

12. Dokuchaev, N.: Maximin investment problems for discounted and total wealth. IMA J. Manag. Math. 19(1), 63-74 (2008)

13. Föllmer, H., Gundel, A.: Robust projections in the class of martingale measures. Ill. J. Math. 50, 439-472 (2006)

14. Friedman, A.: Stochastic Differential Equations and Applications, vol. I-II. Academic Press, New York (1975)

15. Gundel, A.: Robust utility maximization for complete and incomplete market models. Finance Stoch. 9, 151-176 (2005)

16. Hernández-Hernández, D., Schied, A.: Robust utility maximization in a stochastic factor model. Stat. Decis. 24, 109-125 (2006)

17. Hernández-Hernández, D., Schied, A.: A control approach to robust utility maximization with logarithmic utility and time-consistent penalties. Stoch. Process. Appl. 117, 980-1000 (2007)

18. Henry, D.: Geometric Theory of Semilinear Parabolic Equations. Springer, Berlin (1981)

19. Huber, P.J.: Robust Statistics. Wiley, New York (1981)

20. Kazamaki, N.: Continuous Exponential Martingales and BMO. Lecture Notes in Mathematics, vol. 1579. Springer, Berlin (1994)

21. Kobylanski, M.: Backward stochastic differential equations and partial differential equations with quadratic growth. Ann. Appl. Probab. 28, 558-602 (2000)

22. Korn, R., Menkens, O.: Worst-case scenario portfolio optimization: a new stochastic control approach. Math. Methods Oper. Res. 62, 123-140 (2005)

23. Ladyzhenskaya, O.A., Solonnikov, V.A., Ural'tseva, N.N.: Linear and Quasi-Linear Equations of Parabolic Type. American Mathematical Society, Providence (1988)

24. Lazrieva, N., Toronjadze, T.: Optimal robust mean-variance hedging in incomplete financial markets. J. Math. Sci. (N.Y.) 153(3), 262-290 (2008)

25. Liptser, R.Sh., Shiryayev, A.N.: Martingale Theory. Nauka, Moscow (1986)

26. Mania, M., Santacroce, M.: Exponential hedging under partial information. Finance Stoch. 14, 419448 (2010)

27. Matoussi, A., Possamai, D., Zhou, C.: Robust utility maximization in non-dominated models with 2BSDEs. Preprint (2012). arXiv:1201.0769

28. Nisio, M.: Stochastic differential games and viscosity solutions of Isaacs equations. Nagoya Math. J. 110, 163-184 (1988)

29. Quenez, M.-C.: Optimal portfolio in a multiple-priors model. In: Dalang, R.C., Dozzi, M., Russo, F. (eds.) Seminar on Stochastic Analysis, Random Fields and Applications IV. Progr. Probab., vol. 58, pp. 291-321. Birkhäuser, Basel (2004)

30. Schied, A.: Robust optimal control for a consumption-investment problem. Math. Methods Oper. Res. 67, 1-20 (2008)

31. Soner, H.M., Touzi, N., Zhang, J.: Wellposedness of second order backward SDEs. Probab. Theory Relat. Fields 153, 149-190 (2012)

32. Talay, D., Zheng, Z.: Worst case model risk management. Finance Stoch. 6, 517-537 (2002)

33. Tevzadze, R.: Solvability of backward stochastic differential equations with quadratic growth. Stoch. Process. Appl. 118, 503-515 (2008)

34. Touzi, N.: Direct characterization of the value of super-replication under stochastic volatility and portfolio constraints. Stoch. Process. Appl. 88, 305-328 (2000)

35. Warga, J.: Optimal Control of Differential and Functional Equations. Academic Press, New York (1976) 